\title{
Magnetic nanoparticles as many-spin systems
}

\author{
H. Kachkachi ${ }^{a}$ and D. A. Garanin ${ }^{b}$ \\ ${ }^{a}$ Laboratoire de Magnétisme et d'Optique, Université de Versailles St. Quentin, \\ 45 av. des Etats-Unis, 78035 Versailles, France \\ ${ }^{\mathrm{b}}$ Institut für Physik, Johannes-Gutenberg-Universität, D-55099 Mainz, Germany
}

\begin{abstract}
We present a review of recent advances in the study of many-body effects in magnetic nanoparticles. Considering classical spins on a lattice coupled by the exchange interaction in the presence of the bulk and surface anisotropy, we investigate the effects of finite size, free boundaries, and surface anisotropy on the average and local magnetization for zero and finite temperatures and magnetic fields. Superparamagnetism of magnetic particles necessitates introducing two different, induced and intrinsic, magnetizations. We check the validity of the much used relation between them within different theoretical models. We show that the competition between the exchange and surface anisotropy leads to spin canting dependent on the orientation of the average magnetization with respect to the crystallographic axes and thus to a second-order effective anisotropy of the particle. We have also investigated the switching mechanism of the magnetization upon varying the surface anisotropy constant. Some cases of more realistic particles are also dealt with.
\end{abstract}

Key words: Fine particle systems, surface anisotropy, classical spin models PACS: 75.50.Tt - 75.30.Pd - 75.10.Hk

\section{Introduction}

Magnetic nanoparticles, or nanoscale magnetic systems, have generated continuous interest since late 1940s as the investigation of their properties turned out to be challenging from both scientific and technological point of view. In 1949, in a pioneering work [1], Néel set the pace towards understanding of the magnetic behavior of nanoparticles, leading to an important development of fundamental theories of magnetism and modeling of magnetic materials, as well as remarkable technological advances, e.g., in the area of information storage and data processing, fostering the development of magnetorecording media with increasingly higher densities. Nanoparticles, as compared to bulk

Preprint submitted to Elsevier Science 30 October 2018 
materials, possess very important novel properties such as enhanced remanence and giant coercivity, as well as exponentially slow relaxation at low temperature due to anisotropy barriers, which ensures great stability of the information stored. However, nanoparticles become superparamagnetic [1] at finite temperatures for very small sizes, and this is an impairment to the information storage. On the other hand, discovery of superparamagnetism that results from thermally activated crossing of the anisotropy energy barrier by the magnetic moment of the particle opened a rich area to the application of nonequilibrium statistical mechanics. There are mainly two types of nanoparticle samples: i) Assemblies of, e.g., cobalt, nickel or maghemite, nanoparticles with volume distribution and randomly oriented axes of magnetocrystalline anisotropy; ii) Isolated single particles of cobalt or nickel that can be probed by the micro-SQUID technique [2]. While most of the experiments have been done on assemblies, isolated particles are more important both as units of information storage and as a physical system.

In the investigation of the static properties of magnetic nanoparticles a great deal of work up to date has been based on the Monte Carlo (MC) technique. In addition to numerous simulations of the Ising model, this technique has been used with the more adequate classical Heisenberg model to simulate idealized isotropic models with simple cubic (sc) lattice and spherical shape in Ref. [3]. Magnetic nanoparticles with realistic lattice structure were recently simulated in Ref. [4] taking into account the surface anisotropy (SA) and DDI.

On the other hand, in most of theoretical approaches to the dynamics of a small magnetic particle the latter is considered as a single magnetic moment. This is the one-spin approximation that is only valid for particles that are not too large and thus are single-domain, and not too small to be free from surface effects. Letting apart spin tunneling (see, e.g., Ref. [5]) that becomes important for extremely small sizes such as those of molecular magnets, the magnetic moment can overcome the anisotropy-energy barrier and thus reverse its direction, at least in two ways ${ }^{1}$ : Either under applied magnetic field that suppresses the barrier, or via thermal fluctuations. The former, at zero temperature, is well described for particles with the uniaxial anisotropy by the Stoner-Wohlfarth model [7]. Thermally activated crossing of the energy barrier is described by the Néel-Brown model [8] and its extensions (see, e.g., Refs. [9]). At elevated temperatures, rotation of the magnetization in materials with strong anisotropy is always accompanied by changing its magnitude. This results in a shrinking of the Stoner-Wohlfarth astroid as described by the modified Landau theory [10], and (qualitatively) confirmed by experiments $[2]$.

1 It has quite recently been shown [6], experimentally and theoretically, that efficient magnetization switching can be triggered by transverse field pulses of a duration that is half the precession period. 
Both Stoner-Wohlfarth and Néel-Brown models have been confirmed by experiments on individual cobalt particles [2]. However for magnetic particles with strong surface anisotropy magnetization switching occurs as the result of successive switching of individual (or clusters of) spins inside the particle [11]. Such deviations from the one-spin approximation have been observed in metallic particles [14], [16], and ferrite particles [12], [13]. Deviations from the one-spin approximation and temperature effects lead to the absence of magnetization saturation at high fields [14], [15], [16], shifted hysteresis loops after cooling in field, and field dependence of the magnetization at very low temperatures. The latter effect has been clearly identified in dilute assemblies of maghemite particles [17] of $4 \mathrm{~nm}$ in diameter. In addition, aging effects have been observed in single particles of cobalt and have been attributed to the oxidation of the sample surface into antiferromagnetic $\mathrm{CoO}$ (see [2] and references therein.). It was argued that the magnetization reversal of a ferromagnetic particle with antiferromagnetic shell is governed by two mechanisms that are supposed to result from the spin frustration at the core-shell interface of the particle. Some of the above-mentioned novel features are most likely due to magnetic disorder at the surface which induces a canting of spins inside the particle, or in other words, an inhomogeneous magnetic state. This effect was first observed with the help of Mössbauer spectroscopy by Coey [18] and later by Morrish and Haneda (see [19] for a review), and later by Prené et al. [20] (see also the recent article [21]). To sum up, the picture of a single-domain magnetic particle with all spins pointing into the same direction is no longer valid when one considers the effect of misaligned spins on the surface, which makes up to $50 \%$ of the total volume in a particle of $4 \mathrm{~nm}$ in diameter.

One of our goals is thus is to understand the effect of surfaces on the thermodynamics and magnetization profiles in small systems, and subsequently upon their dynamics. This requires a microscopic approach to account for the local environment inside the particle, microscopic interactions such as spin-spin exchange, DDI, and the magneto-crystalline bulk and surface anisotropy. As this task is difficult owing to the large number of degrees of freedom involved, one has to gain a sufficient understanding of static properties before proceeding to the dynamics. There are three main effects that distinguish magnetic particles from bulk magnets and that were investigated in a series of our recent publications:

(1) Finite-size effect in isotropic magnetic particles with idealized periodic boundary conditions. The spin-wave spectrum of such particles is discrete and there is the mode with $\mathbf{k}=0$ that corresponds to the global rotation of the particle. As a result, the standard spin-wave theory fails and one has to distinguish between induced and intrinsic magnetizations.

(2) Boundary effect, i.e., pure effect of the free boundary conditions at the surface in the absence of the surface anisotropy. This effect leads to the decrease of the particle's intrinsic magnetization and it makes the latter 
inhomogeneous at $T \neq 0$.

(3) Effect of surface anisotropy that changes the ground state of the particle and makes the intrinsic magnetization inhomogeneous even at $T=0$.

This paper is organized as follows: In Sec. 2 we describe the Hamiltonian and introduce the basic notions of the induced and the intrinsic magnetizations. In Sec. 3, we first study inhomogeneities in small magnetic particles of a box shape induced by pure boundary effects in the absence of surface anisotropy, i.e., the effect of free boundary conditions (fbc), and compare their influence with that of the finite-size effects in an idealized model with periodic boundary conditions (pbc). We consider spins as $D$-component classical vectors. At first we present analytical and numerical results in the whole range of temperatures in the limit $D \rightarrow \infty$ where the problem simplifies. Then for the classical Heisenberg model, $D=3$, at low temperatures we formulate the modified spin-wave theory accounting for the global rotation of the particle's magnetization. The results are compared with those of our MC simulations. In Sec. 4 we consider round-shaped systems and include surface anisotropy. In the case of the SA much weaker than the exchange interaction we study the problem perturbatively in small deviations from the perfectly ordered, collinear state. Then we investigate the hysteretic properties and the behavior of the magnetization as a function of temperature and applied field by MC simulations. The last section summarizes the results and points out open problems.

\section{Basic relations}

\subsection{The Hamiltonian}

Within the classical approximation it is convenient to represent the atomic spin as the three-component spin vector $\mathbf{s}_{i}$ of unit length on the lattice site $i$. We will consider the Hamiltonian that in general includes the exchange interaction, magneto-crystalline anisotropic energy, Zeeman energy, and the energy of dipolar interactions (DDI)

$$
\mathcal{H}=-\frac{1}{2} \sum_{i j} J_{i j} \mathbf{s}_{i} \cdot \mathbf{s}_{j}-\mu_{0} \mathbf{H} \cdot \sum_{i} \mathbf{s}_{i}+\mathcal{H}_{\mathrm{an}}+\mathcal{H}_{\mathrm{DDI}}
$$

where $\mu_{0}=g \mu_{B} S$ and $S$ is the value of the atomic spin. For materials with uniaxial anisotropy $\mathcal{H}_{\text {an }}$ in Eq. (1) reads

$$
\mathcal{H}_{\text {an }}^{(\text {uni })}=-\sum_{i} K_{i}\left(\mathbf{s}_{i} \cdot \mathbf{e}_{i}\right)^{2}
$$


with easy axis $\mathbf{e}_{i}$ and constant $K_{i}>0$. This anisotropy model can be used to describe the surface effect if one attributes the same easy axis and the same anisotropy constant $K_{c}$ for all core spins and different easy axes and anisotropy constants to surface spins. Within the simplest transverse surface anisotropy (TSA) model all surface spins have the same anisotropy constant $K_{s}$, whereas their easy axes are perpendicular to the surface, see. e.g., Refs. [22,23,24] . More realistic is Néel's surface anisotropy (NSA) model [25],

$$
\mathcal{H}_{\mathrm{an}}^{(\mathrm{NSA})}=-L \sum_{i} \sum_{j=1}^{z_{i}}\left(\mathbf{s}_{i} \cdot \mathbf{e}_{i j}\right)^{2}, \quad \mathbf{e}_{i j} \equiv \mathbf{r}_{i j} / r_{i j}, \quad \mathbf{r}_{i j} \equiv \mathbf{r}_{i}-\mathbf{r}_{j}
$$

where $z_{i}$ is the coordination number of site $i$ that for the surface atoms is smaller than the bulk value $z$ and $\mathbf{e}_{i j}$ is the unit vector connecting the site $i$ to its nearest neighbors $j$. One can check that for the simple cubic (sc) lattice the contributions from the bulk spins in $(3) \sim \mathbf{s}_{i}^{2}=1$ are irrelevant constants.

For materials with magneto-crystalline cubic anisotropy $\mathcal{H}_{\text {an }}$ reads

$$
\mathcal{H}_{\mathrm{an}}^{(4)}=-K^{(4)} \sum_{i}\left(s_{i x}^{4}+s_{i y}^{4}+s_{i z}^{4}\right)
$$

For $K^{(4)}>0$, the energy $\mathcal{H}_{\text {an }}^{(4)}$ has minima for six orientations of the type [100] and maxima for eight orientations of type [111].

If one discards $\mathcal{H}_{\mathrm{an}}^{(4)}$ and $\mathcal{H}_{\mathrm{DDI}}$, the Hamiltonian (1) can be generalized for $D$-component spin vectors. This is useful as in the limit $D \rightarrow \infty$ the problem simplifies while retaining important physics, see below.

\subsection{Magnetization of finite systems}

Magnetic particles of finite size do not show magnetic ordering at nonzero temperatures at $H=0$ as the global magnetization of the particle can assume all possible directions (superparamagnetism). It is thus convenient to define two magnetizations, $m$ and $M$, the first being the magnetization induced by the magnetic field and the second being a measure of the short-range order in the particle. Omitting the factor $\mu_{0}$ that can be restored later, we first define the magnetization of a microscopic spin configuration

$$
\mathbf{M}=\frac{1}{\mathcal{N}} \sum_{i} \mathbf{s}_{i}
$$


where $\mathcal{N}$ is the number of magnetic atoms in the system. The thermodynamic average of $\mathbf{M}$ yields what we call the induced magnetization

$$
\mathbf{m}=\langle\mathbf{M}\rangle=\frac{1}{\mathcal{N}} \sum_{i}\left\langle\mathbf{s}_{i}\right\rangle
$$

The intrinsic magnetization is related to the spin correlation function:

$$
M=\sqrt{\left\langle\mathbf{M}^{2}\right\rangle}=\sqrt{\left\langle\left(\frac{1}{\mathcal{N}} \sum_{i} \mathbf{s}_{i}\right)^{2}\right\rangle}=\frac{1}{\mathcal{N}} \sqrt{\sum_{i j}\left\langle\mathbf{s}_{i} \cdot \mathbf{s}_{j}\right\rangle} .
$$

If the temperature is low and there is no surface anisotropy, all spins in the particle are bound together by the exchange interaction and $\mathbf{M}$ behaves as a rigid "giant spin", $|\mathbf{M}| \cong M \cong 1$. If a magnetic field $\mathbf{H}$ is applied, $\mathbf{M}$ exhibits an average in the direction of $\mathbf{H}$, which leads to a nonzero value of the induced magnetization $\mathbf{m}$. For isotropic $D$-component vector models (including the Ising model, $D=1$ ) [26] the latter is given by the well known formula

$$
m=M B_{D}(M x), \quad x \equiv \mathcal{N} H / T,
$$

where $B_{D}(x)$ is the Langevin function $\left[B_{3}(x)=\operatorname{coth} x-1 / x\right.$ for the isotropic Heisenberg model and $B_{1}(x)=\tanh x$ for the Ising model]. An important question is whether Eq. (8) remains valid at elevated temperatures where $M=M(T, H)$. We have shown that the superparamagnetic relation, Eq. (8), becomes exact for $D \rightarrow \infty$ but otherwise it contradicts the exact relation

$$
M^{2}=m^{2}+\frac{d m}{d x}+\frac{(D-1) m}{x}
$$

One also can introduce the local intrinsic magnetization $M_{i}$ according to

$$
M_{i}=\frac{1}{M}\left\langle\mathbf{s}_{i} \cdot \frac{1}{\mathcal{N}} \sum_{j} \mathbf{s}_{j}\right\rangle, \quad \frac{1}{\mathcal{N}} \sum_{i} M_{i}=M
$$

This quantity is smaller near the boundaries of the particle than in the core for the model with fbc because of boundary effects at $T>0$. 


\section{Nanoparticle as a multi-spin system: finite-size vs boundary ef- fects}

Finite-size magnetic systems with free boundary conditions (fbc) present a spatially inhomogeneous many-body problem. In this section we shall only deal with finite-size versus boundary effects, leaving the more profound effects of the surface anisotropy for the next section. One of the interesting problems here is the interplay between boundary effects due to fbc and the "pure" finitesize effects. In systems of hypercubic shape, the latter can be singled out by using artificial periodic boundary conditions (pbc). The standard mean-field approximation (MFA) and spin-wave theory (SWT) are inappropriate for finite systems because of the Goldstone mode associated with the global rotation of the magnetic moment in zero field. Appropriate improvements include the so-called $D \rightarrow \infty$ model (see Sec. 3.1) operating at all temperatures and the modified spin-wave theory for finite magnets at low temperatures (see Sec. 3.2). Also the MC routine should incorporate global rotations of spins, in addition to the Metropolis algorithm of individual spin rotations.

\section{1 $D \rightarrow \infty$ model}

\subsubsection{The model}

One can improve upon the MFA by taking into account correlations in a wide temperature range for bulk and finite magnets by replacing 3-component spin vectors in (1) by $D$-component ones and taking the limit $D \rightarrow \infty$. This model was introduced by Stanley [26] who showed that in the bulk its partition function coincides with that of the exactly solvable spherical model (SM) [27]. On the other hand, for spatially inhomogeneous and anisotropic systems the $D \rightarrow \infty$ model is the only physically acceptable model of both (see, e.g., Refs. [28]). So far, the $D \rightarrow \infty$ model was only applied to spatially inhomogeneous systems in the plane geometry [28]. In Ref. [10] we extended it to finite boxshaped magnetic systems with free and periodic boundary conditions. In the MFA the Curie temperature of the $D$-component model is $T_{c}^{\mathrm{MFA}}=J_{0} / D$, where $J_{0}$ is the zero Fourier component of $J_{i j}$. It is convenient to use $T_{c}^{\mathrm{MFA}}$ as the energy scale and introduce the dimensionless variables

$$
\theta \equiv T / T_{c}^{\mathrm{MFA}}, \quad \mathbf{h} \equiv \mathbf{H} / J_{0}, \quad \lambda_{i j} \equiv J_{i j} / J_{0}
$$

For the nearest-neighbor (nn) interaction $J_{i j}$ with $z$ neighbors, $\lambda_{i j}$ is equal to $1 / z$ if sites $i$ and $j$ are nearest neighbors and zero otherwise. In the bulk the $D \rightarrow \infty$ model is described by two coupled nonlinear equations for the 
magnetization $m$ and the so called gap parameter $G$ :

$$
m=\frac{h G}{1-G}, \quad m^{2}+\theta G P(G)=1, \quad P(G)=\int \frac{d^{3} \mathbf{k}}{(2 \pi)^{3}} \frac{1}{1-G \lambda_{\mathbf{k}}},
$$

where $P(G)$ is the lattice Green function and $\lambda_{\mathbf{k}}=\left(\cos k_{x}+\cos k_{y}+\cos k_{z}\right) / 3$ is the Fourier transform of $\lambda_{i j}$. The Curie temperature is defined by $G=1$ and is $T_{c}=T_{c}^{\mathrm{MFA}} / W$, i.e., $\theta_{c}=1 / W$, where $W \equiv P(0)$ is the Watson integral ( $W=1.51639$ for the sc lattice). The system of equations describing the inhomogeneous $D \rightarrow \infty$ model can be obtained using the diagram technique for classical spin systems $[29,30]$ in the limit $D \rightarrow \infty$ and generalising the results of Ref. [28] for spatially inhomogeneous systems to include the magnetic field $\mathbf{h}=h \mathbf{e}_{z}$. This is a system of equations for the average magnetization $m_{i} \equiv\left\langle s_{z i}\right\rangle$ directed along the field, gap parameter $G_{i}$, and correlation functions for the remaining "transverse" spin components labeled by $\alpha \geq 2$, i.e., $s_{i j} \equiv$ $D\left\langle s_{\alpha i} s_{\alpha j}\right\rangle$ (all transverse correlation functions are the same). This system of equations has the form

$$
\sum_{j} \mathcal{D}_{i j} m_{j}=h, \quad \sum_{j} \mathcal{D}_{i j} s_{j l}=\theta \delta_{i l}, \quad s_{i i}+m_{i}^{2}=1
$$

where $\mathcal{D}_{i j} \equiv G_{i}^{-1} \delta_{i j}-\lambda_{i j}$ is the Dyson matrix and $\delta_{i l}$ is the Kronecker symbol. Solving this system of equations consists in determining $m_{i}$ and $s_{i j}$ as functions of $G_{i}$ from the first two linear equations and inserting the solutions into the third nonlinear equation (the constraint equation) that leads to a system of nonlinear equations for all $G_{i}$ that is in general subject to numerical solution. Combining Eqs. (13) results in $m^{2}+\theta m /(\mathcal{N} h)-M^{2}=0$ that yields

$$
m=M \frac{2 \mathcal{N} M h / \theta}{1+\sqrt{1+(2 \mathcal{N} M h / \theta)^{2}}}=M B_{\infty}(\mathcal{N} M H / T),
$$

where

$$
M=\sqrt{\mathbf{m}^{2}+\frac{1}{\mathcal{N}^{2}} \sum_{i j} s_{i j}}, \quad B_{\infty}(\xi)=\frac{2 \xi / D}{1+\sqrt{1+(2 \xi / D)^{2}}}
$$

and is $B_{\infty}(\xi)$ the Langevin function for $D \gg 1$. Alternatively Eq. (14) can be derived from Eq. (9) replacing $D-1 \Rightarrow D$ and neglecting $d m / d x$ in the limit $D \rightarrow \infty$ and then solving the resulting algebraic equation for $m$. One can find in the literature formulae of the type $m=M_{s} B\left(\mathcal{N} M_{s} H / T\right)$, where the saturation magnetization $M_{s}$ is usually associated with the bulk magnetization at a given temperature (see, e.g., Refs. [31]). In our case, Eq. (14) is exact and 

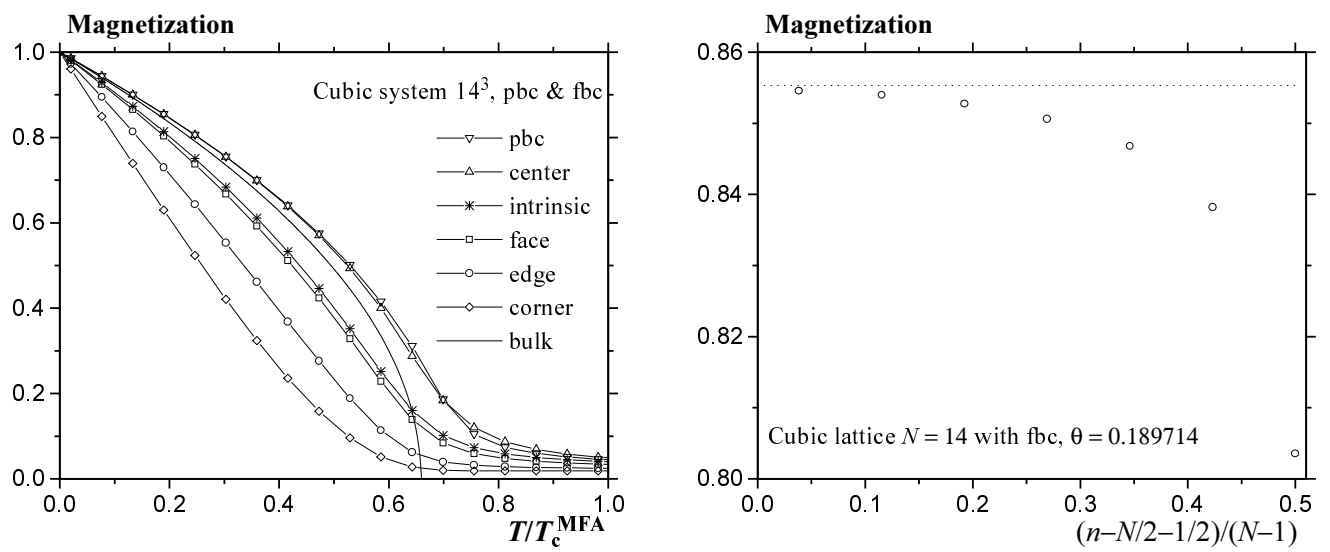

Fig. 1. Left: Temperature dependence of the intrinsic magnetization $M$, Eq. (15), and local magnetizations $M_{i}$, Eq. (10 ) in zero field. Right: Long-range magnetization profile in the direction from the center of the cube to the center of a face at temperature $\theta \equiv T / T_{c}^{\mathrm{MFA}}=0.189714$.

$M=M(T, H)$ is defined by Eq. (15). For large sizes $\mathcal{N}$, Eq. (14) describes two distinct field ranges separated at $H \sim H_{V}$ where

$$
H_{V} \equiv \frac{T D}{\mathcal{N} M}
$$

In the range $H \lesssim H_{V}$ the total magnetic moment of the system is disoriented by thermal fluctuations, $m<M$. In the range $H \gtrsim H_{V}$, the total magnetic moment is oriented by the field, $m$ approaches $M$, and both further increase with the field towards saturation $(m=M=1)$ due to the suppression of spin waves in the system. This scenario is inherent to all $O(D)$ models [31].

Having established the superparamagnetic relation, Eq. (14) we are left with the problem of calculating $M(T, H)$. For the pbc the solution becomes homogeneous and one obtains (12) where in $P(G)$ the integral is replaced by a sum over discrete wave vectors $[10]$, whereas $M=\sqrt{m^{2}+\theta G /[\mathcal{N}(1-G)]}$. For the model with fbc analytical solution is only possible at low and high temperatures. At $\theta \ll 1$ small deviations from the collinear state with $M=1$ can be described by the modified SWT for arbitrary $D$, see Sec. 3.2.

\subsubsection{Numerical results and discussion}

The method for solving the $D \rightarrow \infty$ model consists in obtaining the correlation functions $s_{i j}$ and magnetization $m_{i}$ from the first two linear equations in Eq. (13), substituting them into the third equation of Eq. (13), and solving the resulting system of nonlinear equations for the gap parameter $G_{i}$ numerically. Fig. 1 (left) shows the temperature dependence of the intrinsic magnetization 


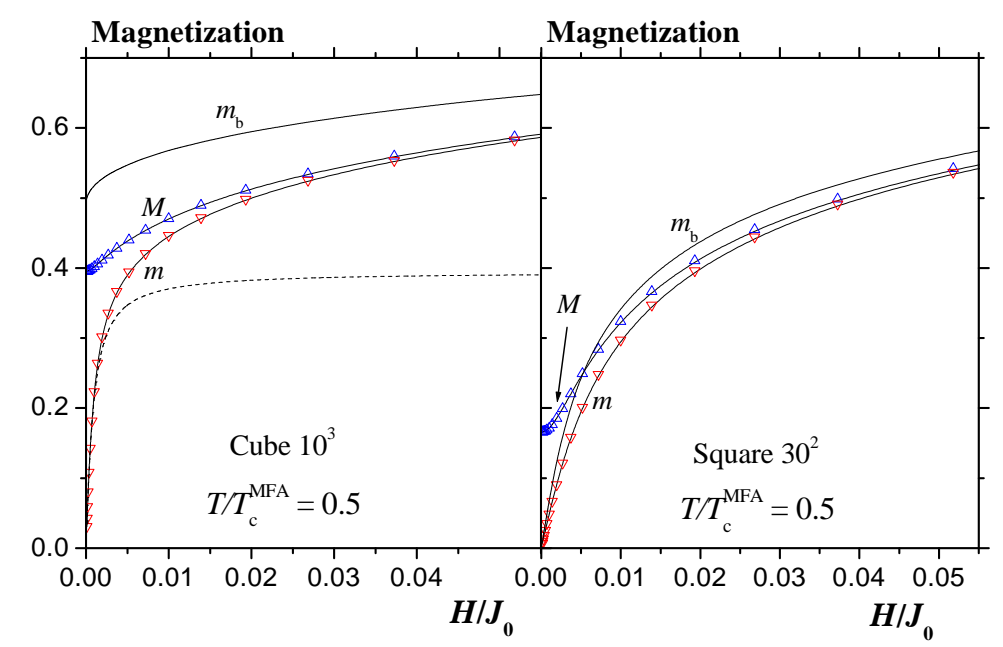

Fig. 2. Field dependence of the intrinsic magnetization $M$ and induced magnetization $m$ for hypercubic lattices with fbc in three and two dimensions. Dashed line is a plot of Eq. (14) in which $M(H, T)$ is replaced by its zero-field value. Bulk magnetization $m_{\mathrm{b}}$ in two and three dimensions is shown by solid lines.

$M$, Eq. (15), and local magnetizations $M_{i}$, Eq. (10), of the $14^{3}$ cubic system with free and periodic boundary conditions in zero field. For periodic boundary conditions, $M$ exceeds the bulk magnetization at all temperatures. In particular, at low temperatures this is in accord with the positive sign of the finite-size correction to the magnetization, Eqs. (24) and (26). Local magnetizations at the center of the faces and edges and those at the corners decrease with temperature much faster than the magnetization at the center. One can see that below the bulk critical temperature $M$ is smaller than the bulk magnetization. This means that the boundary effects suppressing $M$ are stronger than the finite-size effects that increase $M$. This is also seen from the low-temperature expression of $M$ given in Eq. (24), see also Fig. 3. Fig. 1 (right) shows the magnetization profile in the direction from the center of the cube to the center of a face. It is seen that perturbations due to the free boundaries extend deep into the particle, whereas the MFA predicts, on the contrary, a fast approach to a constant magnetization when moving away from the boundary [3]. This is a consequence of the Goldstone mode which renders the correlation length of an isotropic bulk magnet infinite below $T_{c}$. MC simulations of the classical Heisenberg model [3], [4] yield a similar result (see Fig. 14 right).

We have shown that the critical indices for the magnetization at the faces, edges, and corners are higher than the bulk critical index $\beta=1 / 2$ for the present $D=\infty$ model. The critical index at the face $\beta_{1}$ is the most studied surface critical index (see, for a review, Refs. [32]). The exact solution of Bray and Moore [33] for the correlation functions at criticality in the $D=\infty$ model and application of the scaling arguments yield the value $\beta_{1}=1$ (see Table II in [32]). Exact values of the edge and corner magnetization indices, $\beta_{2}$ and $\beta_{3}$, 
seem to be unknown for $D=\infty$. Cardy [34] used the first-order $\varepsilon$-expansion to obtain $\beta_{2}(\alpha)$ for the edge with an arbitrary angle $\alpha$. For $\alpha=\pi / 2$ and $D=\infty$ in three dimensions the result for the edge critical exponent reads $\beta_{2}=$ $13 / 8+O\left(\varepsilon^{2}\right)=1.625+O\left(\varepsilon^{2}\right)$. To estimate the magnetization critical indices in our model we have performed a finite-size-scaling analysis (see, for a review, Ref. [35]) assuming the scaling form $M=N^{-\beta / \nu} F_{M}\left(\tau N^{1 / \nu}\right)$ and plotting the magnetization times $N^{\beta / \nu}$ vs $\tau N^{1 / \nu}$. Here $\nu=1$ is the critical index for the correlation length in the bulk and $\tau \equiv T / T_{c}-1$, where $T_{c}=T_{c}^{\mathrm{MFA}} / W$ is the bulk Curie temperature. Our results for the system with $N=10$ and $N=14$ merge into single "master curves" for $\beta_{1}=0.86, \beta_{2}=1.33$, and $\beta_{3}=1.79$, which have been obtained by fitting $M \propto N^{-\beta / \nu}$ at $T=T_{c}$, i.e., $\theta=\theta_{c}=1 / W$. Note that our value 0.86 for the surface magnetization critical index $\beta_{1}$ is substantially lower than the value $\beta_{1}=1$ following from scaling arguments. This disagreement is probably due to corrections to scaling which could be pronounced for our small linear sizes $N=10$ and 14. A more efficient way for obtaining an accurate value of $\beta_{1}$ is to perform a similar analysis for the semi-infinite model. The latter was considered analytically and numerically for $T \geq T_{c}$ and $H=0$ in [36]. We also mention the Monte Carlo simulations of the Ising model [37] which yield $\beta_{1}=0.80, \beta_{2}=1.28$, and $\beta_{3}=1.77$.

The field dependence of $M$ and $m$ at fixed temperature, as obtained from the numerical solution of Eqs. (13) for cubic and square systems is shown in Fig. 2. Naturally the numerical results for $m$ confirm Eq. (14) which describes both the effect of orientation of the system's magnetization by the field and the increase of $M$ in field. Using the zero-field value of $M$ in Eq. (14) leads to a poor result for $m$ as shown by the dashed curve for the $10^{3}$ system in Fig. 2. The field dependence of the particle's magnetization similar to that shown in Fig. 2 for the cubic system was experimentally obtained for ultrafine cobalt particles in [16], as well as in a number of previous experiments. The curves for the square system in Fig. 2 illustrate the fact that in two dimensions thermal fluctuations are much stronger than in $3 d$, which leads to lower values of both $M$ and $m$ at a given temperature. The bulk magnetization $m_{\mathrm{b}}$ in two dimensions vanishes at zero field and it thus goes below the intrinsic magnetization $M$ in the low-field region.

\subsection{Modified spin-wave theory: Low-temperature properties and the super- paramagnetic relation}

As briefly discussed in Sec. 2, and in more details in the previous section, it is important to investigate the precise relation between the induced magnetization $\mathbf{m}$ of Eq. (6) and intrinsic magnetization $M$ of Eq. (7) for the more realistic Heisenberg model, $D=3$. To do so, we have developed a finite-size spin-wave theory that yields analytical results at low temperatures. We also 
performed simulations with the improved Monte Carlo technique [38].

\subsubsection{Spin-wave theory for finite-size magnetic particles}

In the absence of SA, at low temperatures all spins in the particle are strongly correlated and form a "giant spin" $\mathbf{M}$ defined in Eq. (5) which behaves superparamagnetically. In addition, there are internal spin-wave excitations in the particle that are responsible for $M(H, T)<1$ at $T>0$. These excitations can be described perturbatively in small deviations of individual spins $\mathbf{s}_{i}$ from the global direction of $\mathbf{M}$. Thus we use $\mathbf{M}=\mathcal{M} \mathbf{n}$ with $|\mathbf{n}|=1$ and insert an additional integration over $d \mathbf{M}=\mathcal{M}^{D-1} d \mathcal{M} d \mathbf{n}$ in the partition function,

$$
\mathcal{Z}=\int \mathcal{M}^{D-1} d \mathcal{M} d \mathbf{n} \prod_{i} d \mathbf{s}_{i} \delta\left(\mathbf{M}-\frac{1}{\mathcal{N}} \sum_{i} \mathbf{s}_{i}\right) \mathrm{e}^{-\mathcal{H} / T}
$$

and first integrate over the magnetization magnitude $\mathcal{M}$. Thus we reexpress the vector argument of the $\delta$-function in the coordinate system specified by the direction of the central spin $\mathbf{n}$ :

$$
\delta\left(\mathbf{M}-\frac{1}{\mathcal{N}} \sum_{i} \mathbf{s}_{i}\right)=\delta\left(\mathcal{M}-\frac{1}{\mathcal{N}} \sum_{i}\left(\mathbf{n} \cdot \mathbf{s}_{i}\right)\right) \delta\left(\frac{1}{\mathcal{N}} \sum_{i}\left[\mathbf{s}_{i}-\mathbf{n}\left(\mathbf{n} \cdot \mathbf{s}_{i}\right)\right]\right)
$$

Then after integration over $\mathcal{M}$ one obtains

$$
\mathcal{Z}=\int d \mathbf{n} \mathcal{Z}_{\mathbf{n}}, \quad \mathcal{Z}_{\mathbf{n}}=\int \prod_{i} d \mathbf{s}_{i} \delta\left(\frac{1}{\mathcal{N}} \sum_{i}\left[\mathbf{s}_{i}-\mathbf{n}\left(\mathbf{n} \cdot \mathbf{s}_{i}\right)\right]\right) \mathrm{e}^{-\mathcal{H}_{\mathrm{eff}} / T}
$$

where $\mathcal{Z}_{\mathbf{n}}$ is the partition function for the fixed direction $\mathbf{n}$ and

$$
\mathcal{H}_{\mathrm{eff}}=-(\mathbf{n} \cdot \mathbf{H}) \sum_{i}\left(\mathbf{n} \cdot \mathbf{s}_{i}\right)-\frac{1}{2} \sum_{i j} J_{i j} \mathbf{s}_{i} \cdot \mathbf{s}_{j}-(D-1) T \ln \left[\frac{1}{\mathcal{N}} \sum_{i} \mathbf{n} \cdot \mathbf{s}_{i}\right] .
$$

In Eq. (19), the $\delta$-function says that the sum of all spins does not have a component perpendicular to $\mathbf{M}$. This will lead to the absence of the $\mathbf{k}=0$ component of the transverse spin fluctuations. That is, the global-rotation Goldstone mode that is troublesome in the standard spin-wave theory for finite systems, has been transformed into the integration over the global direction $\mathbf{n}$ in Eq. (19). $\mathcal{Z}_{\mathbf{n}}$ is computed at low temperature by expanding $\mathcal{H}_{\text {eff }}$ up to bilinear terms in the transverse spin components $\Pi_{i}=\mathbf{s}_{i}-\mathbf{n}\left(\mathbf{n} \cdot \mathbf{s}_{i}\right)$ :

$$
\mathcal{H}_{\text {eff }} \cong E_{0}-\mathcal{N} \mathbf{n} \cdot \mathbf{H}+\frac{1}{2} \sum_{i j} A_{i j} \Pi_{i} \cdot \Pi_{j}
$$




$$
A_{i j} \equiv\left[(D-1) T / \mathcal{N}+\mathbf{n} \cdot \mathbf{H}+\sum_{l} J_{i l}\right] \delta_{i j}-J_{i j}
$$

where $E_{0}=-(1 / 2) \sum_{i j} J_{i j}$ is the zero-field ground-state energy. Next, upon computing the resulting Gaussian integrals over $\Pi_{i}^{\alpha}$, one obtains

$$
\mathcal{Z}_{\mathbf{n}} \cong \exp \left(\frac{-E_{0}+\mathcal{N} \mathbf{n} \cdot \mathbf{H}}{T}\right) \mathcal{N}^{D-1}\left[\frac{(2 \pi T)^{\mathcal{N}-1}}{\prod_{k}{ }^{\prime} \frac{A_{k}}{\mathcal{N}}}\right]^{(D-1) / 2}
$$

where for particles of cubic shape

$$
A_{\mathbf{k}}=A_{0}+J_{\mathbf{k}}-J_{0}, \quad A_{0}=\sum_{i} A_{i j}=(D-1) T / \mathcal{N}+\mathbf{n} \cdot \mathbf{H}
$$

The prime on the product in (22) means omitting the $k=0$ mode. Results for particles of arbitrary shape can be found in Ref. [38]. In Eq. (22) the exponential factor corresponds to rigid spins whereas the second factor describes spin-wave corrections. The latter makes the angular dependence of $\mathcal{Z}_{\mathbf{n}}$ more complicated. Differentiating $\mathcal{Z}$ with respect to $H$ yields the induced magnetization $m$, then the intrinsic magnetization $M$ can be obtained from the exact relation (9), and the validity of the superparamagnetic relation (8) can be checked.

\subsubsection{Induced and intrinsic magnetizations, and superparamagnetic relation}

Now we consider particles of cubic shape $\left(\mathcal{N}=N^{3}\right)$ at low temperatures. For both pbc and fbc, we have obtained the following correction to the magnetization in zero field $[10,38]$

$$
M \cong 1-t, \quad t \equiv \frac{D-1}{2} \frac{W_{N} T}{J_{0}}=\frac{D-1}{2 D} W_{N} \theta, \quad W_{N}=\frac{1}{\mathcal{N}} \sum_{\mathbf{k}}^{\prime} \frac{1}{1-\lambda_{\mathbf{k}}}(24)
$$

where $W_{N}$ is the sum without the $\mathbf{k}=0$ term. The results for pbc and $\mathrm{fbc}$ differ only by the values of the discrete wave vectors in Eq. (24) [10]:

$$
k_{\alpha}=\left\{\begin{array}{c}
2 \pi n_{\alpha} / N, \mathrm{pbc} \\
\pi n_{\alpha} / N, \mathrm{fbc}
\end{array}, \quad n_{\alpha}=0,1, \ldots, N-1\right.
$$

where $\alpha=x, y, z$. This subtle difference is responsible for much stronger ther-

mal fluctuations in the fbc model due to boundary effects. The limit $N \rightarrow \infty$ 


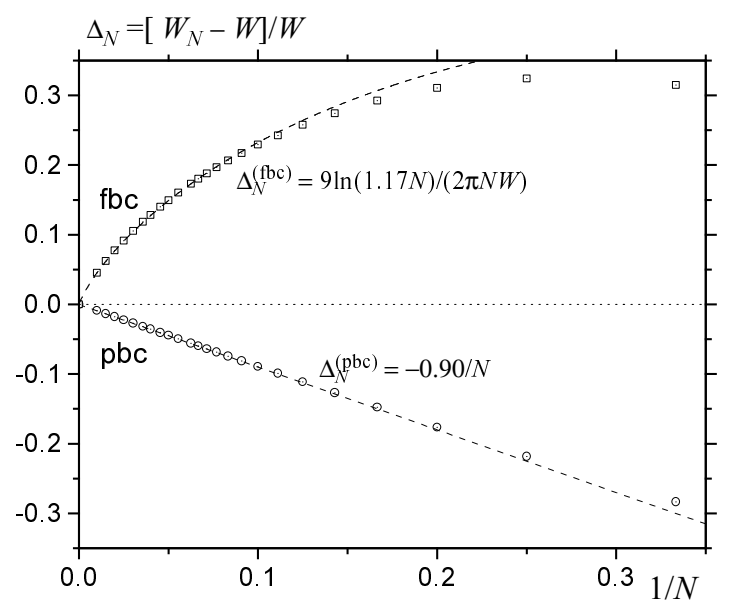

Fig. 3. Lattice sums $W_{N}$ for cubic systems with free and periodic boundary conditions. $W=1.51639$ is the bulk value for the sc lattice.

of $W_{N}$ is the so-called Watson's integral $W=P(0)$ of Eq. (12). The difference between $W_{N}$ and $W$ has different signs for pbc and fbc models [10]

$$
\Delta_{N} \equiv \frac{W_{N}-W}{W} \cong \begin{cases}-\frac{0.90}{N}, & \text { pbc } \\ \frac{9 \ln (1.17 N)}{2 \pi W N}, & \text { fbc }\end{cases}
$$

Therefore, Fig. 3 shows that the coefficient in the linear- $\theta$ term in Eq. (24) is smaller than in the bulk for the pbc system and greater for the fbc system. That is, boundary effects suppress the intrinsic magnetization at low temperatures while finite-size effects lead to its increase. Fig. 3 shows that boundary effects render a larger contribution than the finite-size effects, making the net magnetization well below that of the bulk.

The field dependence of $m$ and $M$ is defined by the expansion of the lattice Green function for small gaps, $1-G \ll 1$

$$
\widetilde{P}_{N}(G)=\frac{1}{\mathcal{N}} \sum_{\mathbf{k}}^{\prime} \frac{1}{1-G \lambda_{\mathbf{k}}} \cong W_{N}-\left\{\begin{array}{cc}
c_{N} N(1-G), & N^{2}(1-G) \ll 1 \\
c_{0} \sqrt{1-G}, & N^{2}(1-G) \gg 1
\end{array}\right.
$$

where for the sc lattice $c_{0}=(2 / \pi)(3 / 2)^{3 / 2}$ and the numerical results for $c_{N}$ can, for $N \gg 1$, be fitted as

$$
c_{N} \cong\left\{\begin{array}{l}
0.384-1.05 / N, \text { pbc } \\
1.90-1.17 / N, \text { fbc }
\end{array}\right.
$$


The spin-wave gap $1-G$ depends on the temperature and field; For $H \ll H_{V}$ [see Eq. (16)] the gap approaches its zero-field value while for $H \gg H_{V}$ one has $1-G \cong h \equiv H / J_{0}$. Thus Eq. (27) defines one more crossover in field, the crossover between its first and second lines at

$$
H_{S} \sim \frac{J_{0}}{\mathcal{N}^{2 / 3}}=\frac{J_{0}}{N^{2}} \gg H_{V}=\frac{T D}{\mathcal{N} M}=\frac{\theta J_{0}}{\mathcal{N} M}
$$

In the field range $H \gg H_{S}$ the discreteness of the lattice can be neglected and the bulk result $\Delta M \sim \sqrt{H}$ is reproduced. In the most interesting region $H \ll H_{S}$ one obtains

$$
M \cong 1-t+2 \alpha x B(x), \quad \alpha \equiv \frac{(D-1) c_{N}}{4 N^{2}}\left(\frac{T}{J_{0}}\right)^{2}
$$

On the other hand, Eq. (30) describes a crossover from the quadratic field dependence of $M$ at low field, $x \ll 1$, to the linear dependence at $x \gg 1$. Note that for $x \gg 1$, where $m \cong M$ and a rigid magnetic moment would saturate, $m$ continues to increase linearly as $m \cong 1-t+2 \alpha x$. This is due to the field dependence of the intrinsic magnetization $M$. At higher fields there is another crossover to the standard spin-wave theory expression for $M$. Approximate expressions for $M$ in the different field ranges are

$$
M \cong 1-t+ \begin{cases}\frac{D-1}{2 D} c_{N}\left(\frac{H N^{2}}{J_{0}}\right)^{2}, & H \ll H_{V} \\ \frac{D-1}{2} c_{N} \frac{N H T}{J_{0}^{2}}, & H_{V} \ll H \ll H_{S} \\ \frac{D-1}{2} c_{0} \frac{T}{J_{0}}\left(\frac{H}{J_{0}}\right)^{1 / 2}, & H_{S} \ll H \ll J_{0} .\end{cases}
$$

A simple analysis shows [38] that the superparamagnetic relation (8) is a very good approximation for not too small systems, $\mathcal{N} \gg 1$ in the whole range below $T_{c}$. The deviation from Eq. ( 8 ) is controlled by the small parameter $\alpha$ of Eq. ( 30 ). Above $T_{c}$, however, deviations from Eq. (8) are large, except for the model with $D \rightarrow \infty$. In the close vicinity of $T_{c}$, there is a crossover to the high-temperature form of Eq. (8) given by the function $B_{\infty}(x)$ of Eq. (15).

The modified SWT developed above can be applied to study inhomogeneities in the fbc model. The local intrinsic magnetization defined by Eq. (10) shows stronger temperature dependence near the boundary than the averaged $M \cong$ $1-t$ of Eq. (24). The biggest effect of the surface is naturally attained at the corners of the cube where $M_{i} \cong 1-8 t$, at $H=0$ [38]. 


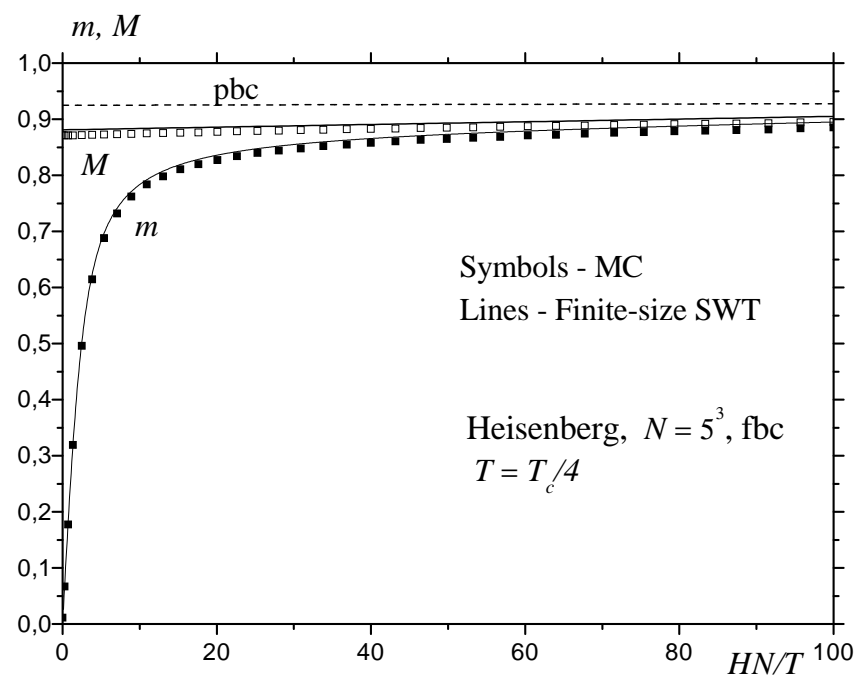

Fig. 4. Comparison of the theoretical and MC results for the field dependences of the magnetizations $M$ and $m$ for the Heisenberg model at $T=T_{c} / 4$.

\subsubsection{MC simulations}

Here we apply our Monte Carlo technique that accounts for the global-rotation Goldstone mode to compute the induced and intrinsic magnetizations, and to investigate the superparamagnetic relation between them, for the Heisenberg model with fbc. Our results for Ising model can be found in [38].

First of all, in Fig. 4 we compare theoretical predictions of our analytical calculations within spin-wave theory for the Heisenberg model with our MC results at $T=T_{c} / 4$, where $T_{c}=0.722 T_{c}^{\mathrm{MFA}}$ is the actual bulk Curie temperature. For the small size $\mathcal{N}=5^{3}$ the square-root field dependence of the magnetization (third line of Eq. (31)) does not arise and finite-size corrections are very important. For $M$ one should use Eq. (30) with $t$ given by Eq. (24) with numerically computed $W_{N}=1.99$ and $c_{N}=1.66$ for the fbc model [cf. Eqs. (26) and (28)]. This yields $t \simeq 0.119$ and $\alpha \simeq 1.20 \times 10^{-4}$. The theoretical dependence $M(H)$ is practically a straight line which goes slightly above the MC points. This small discrepancy can be explained by the fact that the applicability criterion for our analytical method, $t \ll 1$, is not fully satisfied at $T=T_{c} / 4$, and a better agreement is achieved at lower temperatures. For comparison we also plot the theoretical $M(H)$ for the model with periodic boundary conditions. Here one has $W_{N}=1.25$ and $c_{N}=0.20$, thus $t \simeq 0.075$ and $\alpha \simeq 1.45 \times 10^{-5}$, so $M(H)$ goes noticeably higher and with a much smaller slope. The quadratic field dependence of $M$ in the region $x \lesssim 1$ is not seen at this low temperature since the value of $\alpha$ is very small and thus much more accurate MC simulations would be needed. We also plot in Fig. 4 the analytical result for the field dependence of $m$ [38] which favorably compares with our MC data. Fig. 5 (left) shows the intrinsic magnetization $M$ and induced magnetization $m$ versus the scaled field $x \equiv \mathcal{N} H / T$ for different temperatures. 

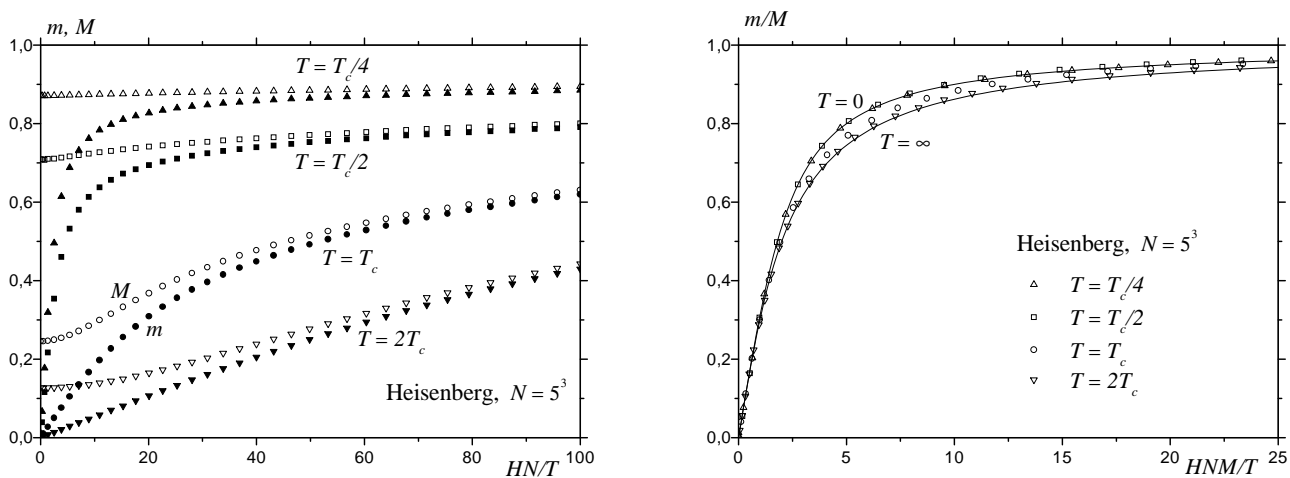

Fig. 5. Left: Field dependence of the intrinsic magnetization $M$ and the induced magnetization $m$ of the Heisenberg model on the sc lattice with fbc for different temperatures. Right: Scaled graph for $m / M$. Theoretical curves $B_{3}(x)=\operatorname{coth} x-1 / x$ for $T \ll T_{c}$ and $B_{\infty}(x)$ for $T \gg T_{c}$ are shown by solid lines.

We see that the particle's magnetic moment is aligned and thus $m \sim M$ for $x \gtrsim 1$, if $T \ll T_{c}$. At $T \gg T_{c}$ the field aligns individual spins, and this requires $H \gtrsim T$, i.e., $x \gtrsim \mathcal{N}$. The quadratic dependence of $M(H)$ at small fields manifests itself strongly at elevated temperatures.

The results of Fig. 5 (right) show that the superparamagnetic relation of Eq. (8) with $M=M(T, H)$ is a very good approximation everywhere below $T_{c}$, for the Heisenberg model. This also holds for the Ising model [38]. On the other hand, above $T_{c}$ Eq. (8) with the function $B_{\infty}(x)$ of Eq. (15) is obeyed. The difference between these limiting expressions decreases with increasing number $D$ of spin components and disappears in the spherical limit $(D \rightarrow \infty)$.

\section{A nanoparticle as a multi-spin system: Effect of surface anisotropy}

Surface anisotropy causes large deviations from the bulk behavior that are much stronger than just the effect of free boundaries. Also SA exerts influence upon the coercive field. Here we first consider magnetic structures and hysteresis loops at zero temperature induced by a strong transverse surface anisotropy (TSA). Then for the more physically plausible Néel's surface anisotropy (NSA) we investigate analytically and numerically its contribution to the effective anisotropic energy of the particle in the case when the NSA is weak in comparison to the exchange. After that we study the effect of the NSA at finite temperatures using the MC technique. In the end of this section we investigate the thermal and spatial dependence of the magnetization of a maghemite particle. 


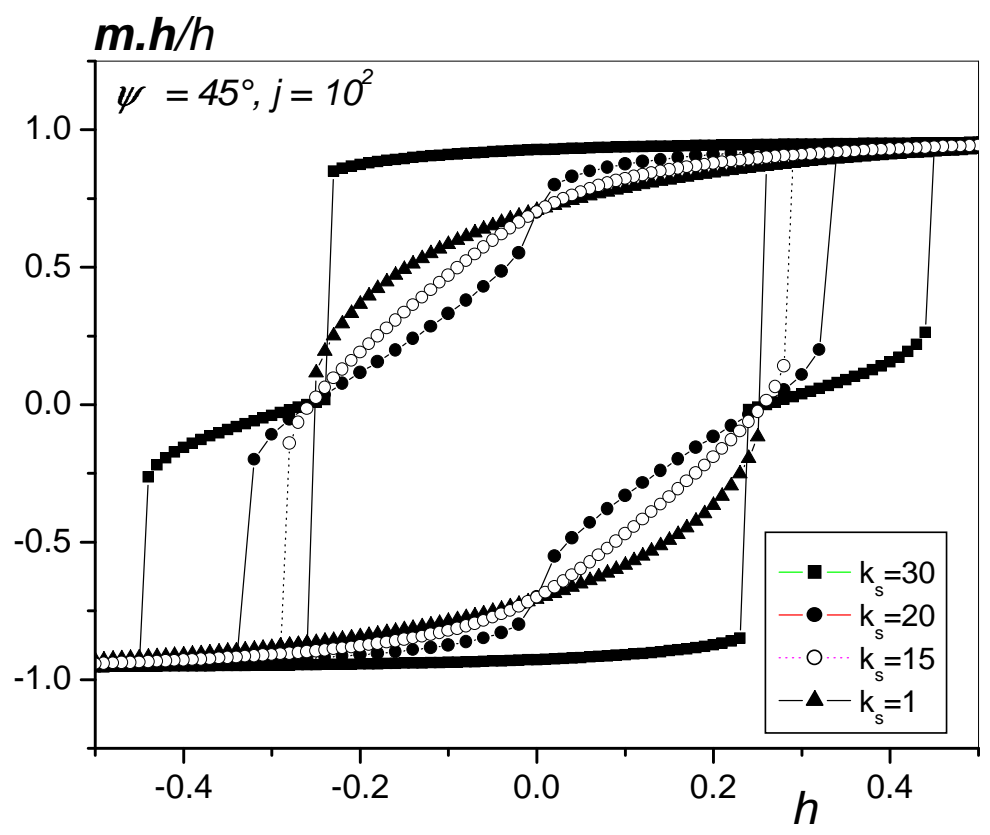

Fig. 6. Hysteresis for $\psi=\pi / 4, j=10^{2}, N=10(\mathcal{N}=360)$ and different $k_{s}$.

\subsection{Magnetic structure and hysteresis at $T=0$ : Transverse surface anisotropy}

Here we study the effect of TSA on the hysteresis loop and the angular dependence of the switching field. We construct an effective Stoner-Wohlfarth (SW) astroid for a single-domain spherical particle with free surfaces, a simple cubic (sc) crystal structure, ferromagnetic exchange $J$, uniaxial anisotropy in the core $K_{c}$, and the TSA of strength $K_{s}$. Using the Hamiltonian (1) without the DDI at $T=0$, we solve the coupled Landau-Lifshitz equations (LLE) for each spin in the particle [11] until a stationary state is attained. In [39] the same method was used for studying hysteresis loops in nanoparticles with a random bulk or surface-only anisotropy. Here we address the question of whether one can still use the simple SW model for a nanoparticle endowed with strong surface effects. We show that it is so as long as $K_{s} \lesssim J$. Otherwise switching of the particle's magnetization occurs via the reversal of clusters of spins, invalidating the simple SW model.

We consider $K_{s}$ and exchange coupling on the surface as free parameters since there are so far no definite experimental estimations of them, whereas the core parameters are taken as for the bulk system. In the sequel, we use the reduced parameters, $j \equiv J / K_{c}, k_{s} \equiv K_{s} / K_{c}$.

Fig. 6 shows that when $k_{s}$ becomes comparable with $j$, the hysteresis loop exhibits multiple jumps, which can be attributed to the switching of different 
spin clusters containing surface spins whose easy axes make the same angle with the field direction. The hysteresis loop is characterized by two field values: One that marks the limit of metastability, called the critical field or the saturation field, and the other that marks the magnetization switching, and is called the switching field or the coercive field. This progressive switching of spins is illustrated in Fig. 7 for simplicity for the non-interacting case.
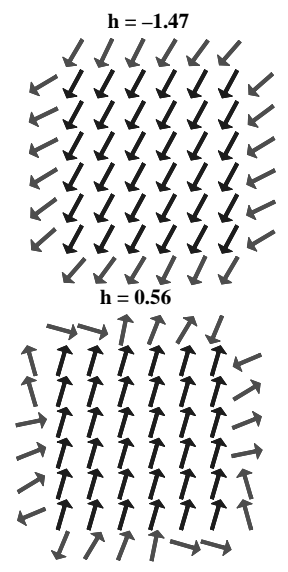
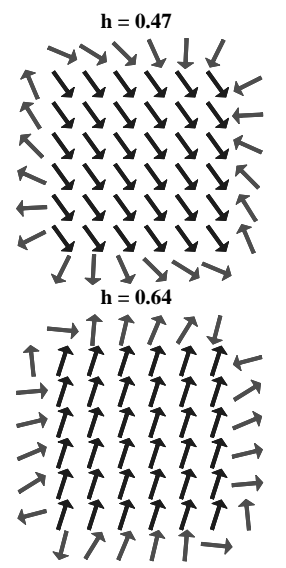
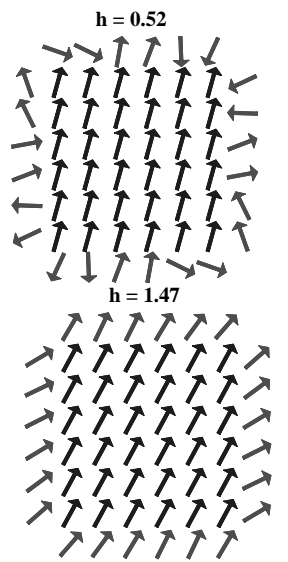

Fig. 7. Magnetic structures in the middle plane of the particle with $\mathcal{N}=360$ in the TSA model for $j=0, k_{s}=1, \psi=\pi / 4$ and different fields.

For small values of $k_{s} / j \sim 0.01$ the TSA model renders hysteresis loops and limit-of-metastability curves that scale with the SW results for all values of the angle $\psi$ between the core easy axis and the applied field, the scaling constant being $\mathcal{N}_{c} / \mathcal{N}<1$, see in Fig. 8 .

For larger values of $k_{s} / j$, but $k_{s} / j \lesssim 0.2$, we still have the same kind of scaling but the corresponding constant now depends on the angle $\psi$ between the core easy axis and field direction. This is reflected by a deformation of the limitof-metastability curve (see Fig. 9a). More precisely, the latter is depressed in the core easy direction and enhanced in the perpendicular direction. However, there is still only one jump in the hysteresis loop implying that the magnetization reversal can be considered as uniform. For $k_{s} / j \gtrsim 1$, there appear multiple steps in the hysteresis loop associated with the switching of spin clusters. It makes the hysteresis loop both qualitatively and quantitatively different from those of the SW model, as the magnetization reversal can no longer be considered as uniform. In addition, in the present case, there are two more new features: the values of the switching field are much higher than in SW model, and more importantly, its behavior as a function of the particle's size is opposite to that of the previous cases (compare Figs. 8a and 9b). More precisely, in this case one finds that this field increases when the particle's size is lowered. This is in agreement with the experimental observations in nanoparticles (see, e.g., [14] for cobalt particles). The whole situation is summarized in Fig. 10 where we plot the critical field $h_{c}$ as a function of $\tilde{k}_{s} \equiv k_{s} / j$ for different values of the surface-to-core ratio of the exchange coupling. For large values of $k_{s}$, 

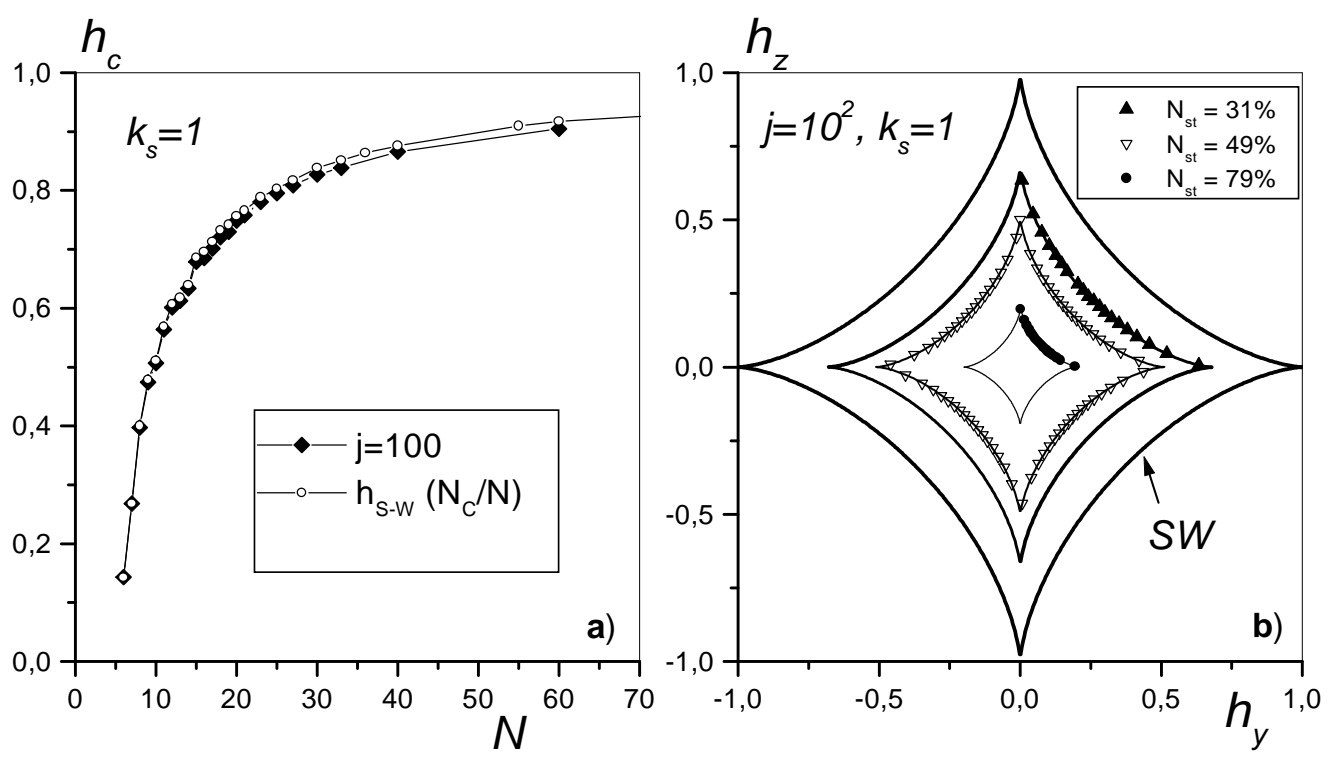

Fig. 8. a) (in diamonds) Switching field for $k_{s}=1, j=10^{2}$ versus the particle's diameter $N$. (in circles) SW switching field multiplied by $N_{c} / \mathcal{N}$. b) Astroid for $k_{s}=1, j=10^{2}$ for different values of the surface-to-volume ratio $N_{s t} \equiv N_{s} / \mathcal{N}$.
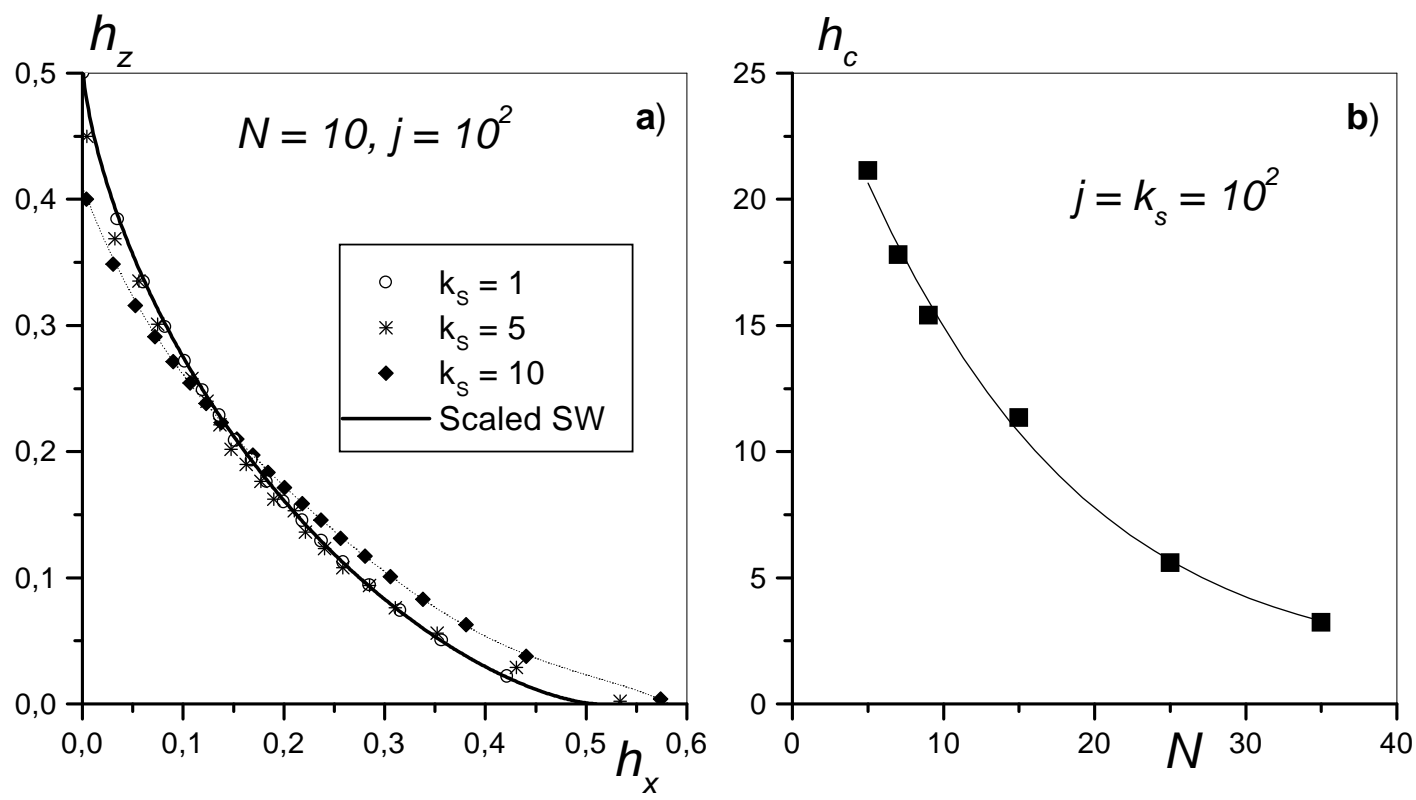

Fig. 9. a) Astroid for $j=10^{2}, \mathcal{N}=360$ and different values of surface anisotropy constant $k_{s}$. The full dark line is the SW astroid scaled with $N_{c} / \mathcal{N}$, but the dotted line is only a guide for the eye. b) Switching field versus the particle's diameter $N$ for $\psi=0, j=k_{s}=10^{2}$. 


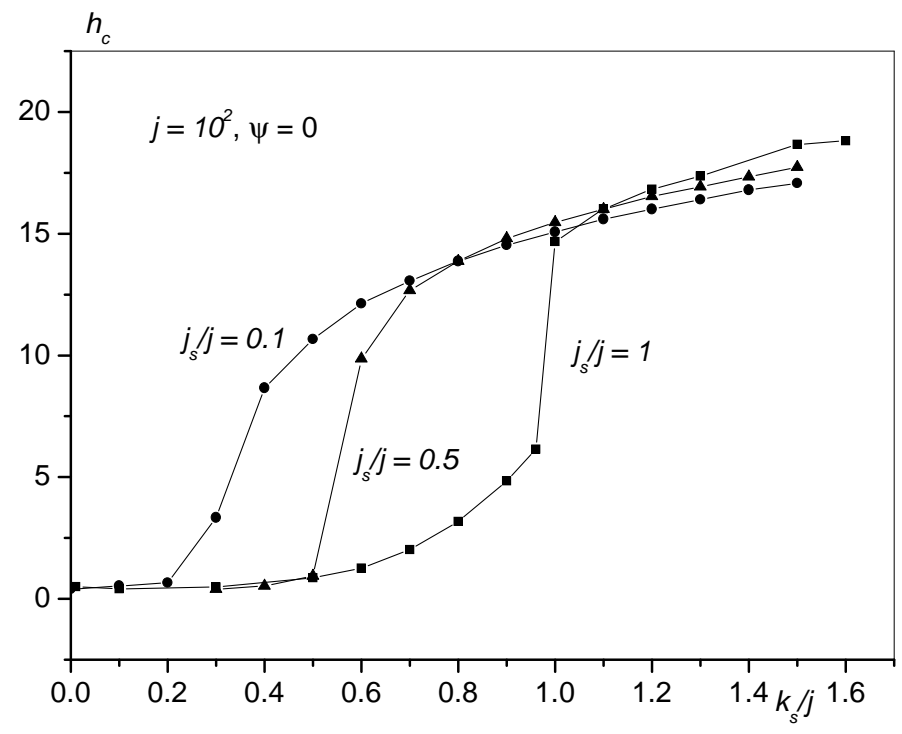

Fig. 10. Critical field versus the surface anisotropy constant for $\psi=0$, and different values of surface-to-core ratio of exchange couplings; $N=10$.

surface spins are aligned along their radial easy axes, and because of strong exchange coupling they also drive core spins in their switching process, which requires a very strong field to be completed. The value of $k_{s}$ where $h_{c}$ jumps up (e.g., $=1$ for $J_{s} / J=1$ ) marks the passage from a regime where scaling with the SW results is possible (either with a $\psi$-dependent or independent coefficient) to the second regime where this scaling is no longer possible because of completely different switching processes.

To estimate $K_{s}$ and the critical field, consider a $4 \mathrm{~nm}$ cobalt particle of fcc crystal structure, for which the lattice spacing is $a=3.554 \AA$, and there are 4 cobalt atoms per unit cell. The (bulk) magneto-crystalline anisotropy is $K_{c} \simeq 3 \times 10^{-17} \mathrm{erg} / \mathrm{spin}$ or $2.7 \times 10^{6} \mathrm{erg} / \mathrm{cm}^{3}$, and the saturation magnetization is $M_{s} \simeq 1422 \mathrm{emu} / \mathrm{cm}^{3}$. The switching field is given by $H_{c}=\left(2 K_{c} / M_{s}\right) h_{c}$. For $\psi=0, \tilde{k}_{s}^{c}=1$ and $h_{c}=15$, so $H_{c} \simeq 6 \mathrm{~T}$. On the other hand, $\tilde{k}_{s}^{c}=1$ means that the effective exchange field experienced by a spin on the surface is of the order of the anisotropy field, i.e. $z S J / 2 \sim 2 K_{s}$. Then using $J \simeq 8 \mathrm{mev}$ we get $K_{s} \simeq 5.22 \times 10^{-14} \mathrm{erg} / \mathrm{spin}$, or using the area per surface spin (approximately $\left.a^{2} / 8\right), K_{s} \simeq 5 \mathrm{erg} / \mathrm{cm}^{2}$. For the case of $\psi=\pi / 4, \tilde{k}_{s}^{c} \simeq 0.2$ and $h_{c} \simeq 0.3$, which leads to $H_{c} \simeq 0.1 \mathrm{~T}$ and $K_{s} \simeq 1.2 \times 10^{-14} \mathrm{erg} / \mathrm{spin}$ or $1.2 \mathrm{erg} / \mathrm{cm}^{2}$.

\subsection{Surface contribution to the energy of magnetic nanoparticles: NSA}

We calculate the contribution of the NSA [25] to the effective anisotropy of magnetic nanoparticles of spherical shape cut out of a simple cubic lattice. The effective anisotropy arises because of deviations of atomic magnetic mo- 


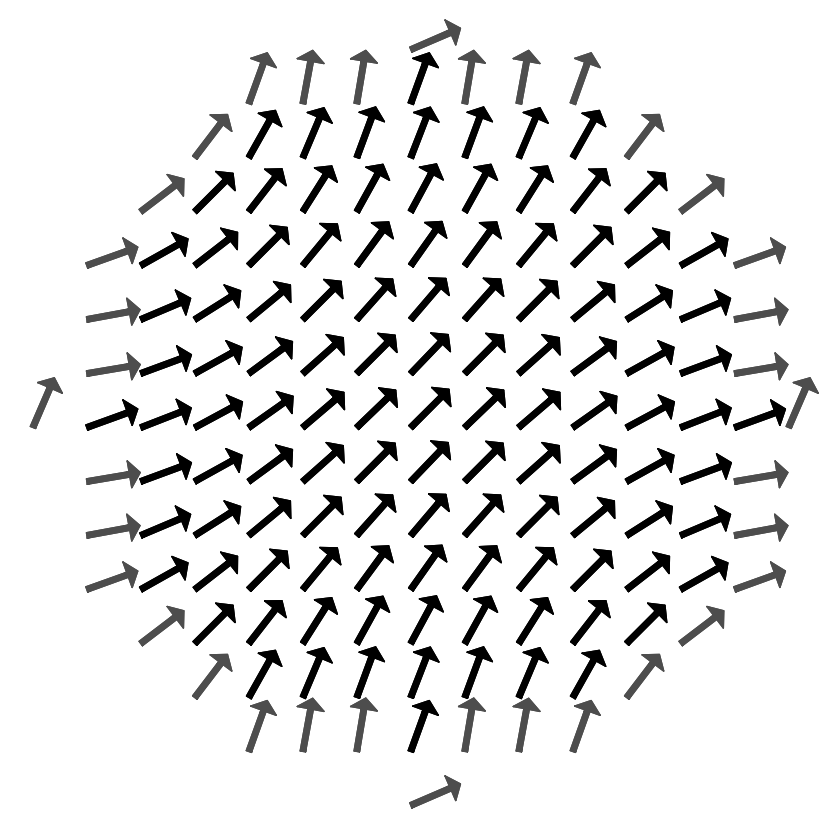

Fig. 11. Magnetic structure in the plane $z=0$ of a spherical nanoparticle of linear size $N=15$ with $L / J=2$ for the global magnetization directed along [110].

ments from collinearity and dependence of the energy on the orientation of the global magnetization with respect to crystallographic directions. We show [40] that the result is second order in the NSA constant, scales with the particle's volume, and has cubic symmetry with preferred directions $[ \pm 1, \pm 1, \pm 1]$.

As shown many times before, as the size of the magnetic particle decreases, surface effects become more and more pronounced. In many cases surface atoms yield a contribution to the anisotropy energy that scales with particle's surface, i.e., to the effective volume anisotropy decreasing with the particle's linear size $R$ as $K_{V \text {,eff }}=K_{V}+K_{S} / R$, as was observed in a number of experiments (see, e.g., Refs. [16], [41]). The $1 / R$ surface contribution to $K_{V \text {,eff }}$ is in accord with the picture of all magnetic atoms tightly bound by the exchange interaction while only surface atoms feel the surface anisotropy. This is definitely true for magnetic films where a huge surface contribution to the effective anisotropy has been observed. The same holds for cobalt nanoclusters of the form of truncated octahedrons [42] where contributions from different faces, edges, and apexes compete, resulting in a nonzero, although significantly reduced, surface contribution to $K_{V \text {,eff }}$.

However, for symmetric particle's shapes such as cubes or spheres, the symmetry leads to vanishing of this first-order contribution. In this case one has to take into account deviations from the collinearity of atomic spins that result from the competition of the surface anisotropy and exchange interaction $J$. The resulting magnetic structures (for the simplified radial SA model) can be found in [39], [43], [11] (see also Fig. 11 for the NSA). In the case $L \gtrsim J$ deviations from collinearity are very strong, and it is difficult, if not impossible, to 

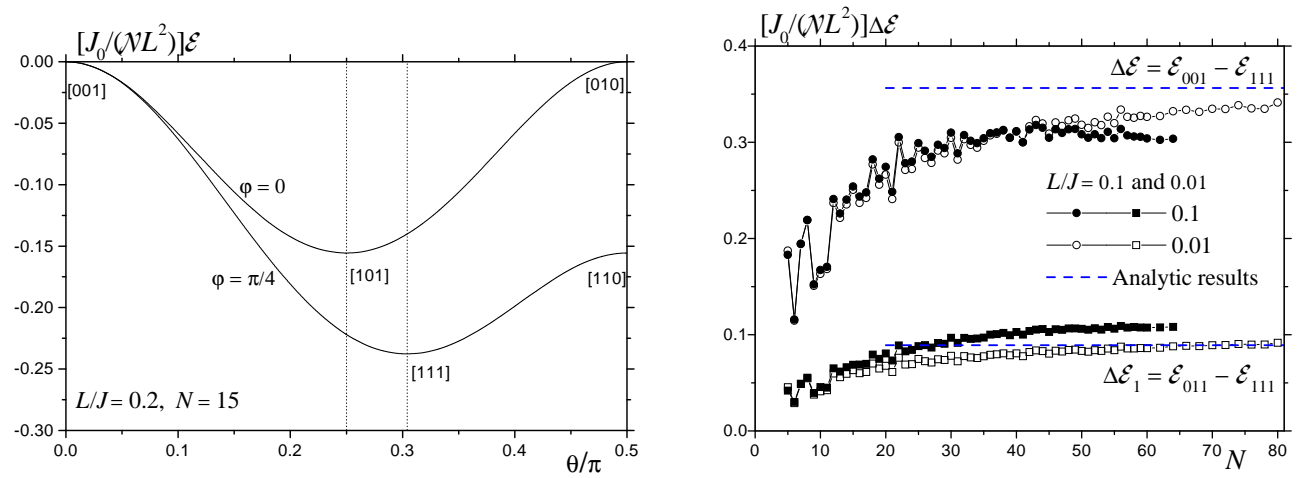

Fig. 12. Left: Effective anisotropy energy of the particle for different orientations of its global magnetization showing cubic symmetry of Eq. (41). Right: Differences of particle's energies between main orientations of the global magnetization vs the particle size in the scaled form for $L / J=0.1$ and 0.01 . The scaling is valid for $N \lesssim J / L$, and its violation for $L / J=0.1$ is seen in the right part of the figure.

characterize the particle by a global magnetization suitable for the definition of the effective anisotropy. For $L \ll J$ the magnetic structure is nearly collinear with small deviations that can be computed perturbatively in $L / J \ll 1$. The global magnetization vector $\mathbf{m}_{0}$ [this is the same as the vector defined in (6)] can be used to define the anisotropic energy of the whole particle. The key point is that deviations from collinearity, and thereby the particle's energy, depend on the orientation of $\mathbf{m}_{0}$, even for a particle of a spherical shape, due to the crystal lattice. To illustrate this idea, we neglect the bulk anisotropy and the DDI in the Hamiltonian (1). For a sc lattice Eq. (3) reduces to

$$
\mathcal{H}_{\mathrm{an}}^{(\mathrm{NSA})}=\sum_{i} \mathcal{H}_{\mathrm{an}, i}^{(\mathrm{NSA})}, \quad \mathcal{H}_{\mathrm{an}, i}^{(\mathrm{NSA})}=\frac{L}{2} \sum_{\alpha=x, y, z} z_{i \alpha} s_{i \alpha}^{2},
$$

where $z_{i \alpha}=0,1,2$ are the numbers of available nearest neighbors of the atom $i$ along the axis $\alpha$. One can see that the NSA is in general biaxial. For $L>0$ and $z_{i \alpha}=0<z_{i \beta}=1<z_{i \gamma}=2$ the $\alpha$-axis is the easy axis and the $\gamma$-axis is the hard axis. If the local magnetic moments $\mathbf{s}_{i}$ are all directed along one of the crystallographic axes $\alpha$, then the anisotropy fields $\mathbf{H}_{A i}=-\partial \mathcal{H}_{A i} / \partial \mathbf{s}_{i}$ are also directed along $\alpha$ and are thus collinear with $\mathbf{s}_{i}$. Hence, at least for $L \ll J$, there are no deviations from collinearity if the global magnetization $\mathbf{m}_{0}$ is directed along one of the crystallographic axes. For other orientations of $\mathbf{m}_{0}$, the vectors $\mathbf{s}_{i}$ and $\mathbf{H}_{A i}$ are not collinear, since then at least two components of $\mathbf{s}_{i}$ are non zero, and the transverse component of $\mathbf{H}_{A i}$ with respect to $\mathbf{s}_{i}$ causes a slight canting of $\mathbf{s}_{i}$ and thereby a deviation from the collinearity of magnetizations on different sites. This adjustment of the magnetization to the surface anisotropy lowers the energy. As we shall see, this effect is strongest for the $[ \pm 1, \pm 1, \pm 1]$ orientations of $\mathbf{m}_{0}$. For both signs of $L$ these are easy orientations, whereas $[ \pm 1,0,0],[0, \pm 1,0]$, and $[0,0, \pm 1]$ are hard orientations. 
To solve the problem numerically, we have to fix the global magnetization of the particle in a desired direction $\nu_{0}\left(\left|\nu_{0}\right|=1\right)$ by using the energy function with a vector Lagrange multiplier $\lambda$ :

$$
\mathcal{F}=\mathcal{H}-\mathcal{N} \lambda \cdot\left(\nu-\nu_{0}\right), \quad \nu \equiv \frac{\sum_{i} \mathbf{s}_{i}}{\left|\sum_{i} \mathbf{s}_{i}\right|} .
$$

To minimize $\mathcal{F}$ we numerically integrate the evolution equations

$$
\begin{aligned}
\dot{\mathbf{s}}_{i} & =-\left[\mathbf{s}_{i} \times\left[\mathbf{s}_{i} \times \mathbf{F}_{i}\right]\right], \quad \mathbf{F}_{i} \equiv-\partial \mathcal{F} / \partial \mathbf{s}_{i} \\
\dot{\lambda} & =\partial \mathcal{F} / \partial \lambda=-\mathcal{N}\left(\nu-\nu_{0}\right) .
\end{aligned}
$$

starting from $\mathbf{s}_{i}=\nu_{0}=\mathbf{m}_{0}$ and $\lambda=0$, until the stationary state is attained and an energy minimum is found. Our numerical results for the magnetic energy of spherical particles as a function of the orientation of the global magnetization are shown in Fig. 12 (left). Fig. 12 (right) shows differences between the basic directions [001], [011], and [111]. It is seen that $\Delta E / \mathcal{N} \rightarrow$ const for $N \rightarrow \infty$ limit, i.e., $\Delta E$ scale with the particle's volume $V \propto \mathcal{N}$.

To analytically solve the problem in the continuous limit, we replace in Eq. (32) the number of nearest neighbors of a surface atom by its average value

$$
z_{i \alpha} \Rightarrow \bar{z}_{i \alpha}=2-\left|n_{\alpha}\right| / \max \left\{\left|n_{x}\right|,\left|n_{y}\right|,\left|n_{z}\right|\right\}
$$

Here $n_{\alpha}$ is the $\alpha$-component of the normal to the surface $\mathbf{n}$. The surface-energy density can then be obtained by dropping the constant term and multiplying Eq. (32) by the surface atomic density $f(\mathbf{n})=\max \left\{\left|n_{x}\right|,\left|n_{y}\right|,\left|n_{z}\right|\right\}$ :

$$
E_{S}(\mathbf{m}, \mathbf{n})=-\frac{L}{2}\left[\left|n_{x}\right| m_{x}^{2}+\left|n_{y}\right| m_{y}^{2}+\left|n_{z}\right| m_{z}^{2}\right] .
$$

At equilibrium the Landau-Lifshitz equation reads

$$
\mathbf{m} \times \mathbf{H}_{\mathrm{eff}}=0, \quad \mathbf{H}_{\mathrm{eff}}=\mathbf{H}_{A}+J \Delta \mathbf{m} .
$$

For small deviations from collinearity one can seek for its solution in the form

$$
\mathbf{m}(\mathbf{r}) \cong \mathbf{m}_{0}+\psi\left(\mathbf{r}, \mathbf{m}_{0}\right), \quad \psi \equiv|\psi| \ll 1
$$

where $\psi$ is the solution of the internal Neumann boundary problem

$$
\Delta \psi=0,\left.\quad \frac{\partial \psi}{\partial r}\right|_{r=R}=\mathbf{f}(\mathbf{m}, \mathbf{n})
$$




$$
\mathbf{f}=-\frac{1}{J}\left[\frac{d E_{S}(\mathbf{m}, \mathbf{n})}{d \mathbf{m}}-\left(\frac{d E_{S}(\mathbf{m}, \mathbf{n})}{d \mathbf{m}} \cdot \mathbf{m}\right) \mathbf{m}\right] .
$$

This equation can be solved with the help of the Green function $G\left(\mathbf{r}, \mathbf{r}^{\prime}\right)$ (see Ref. [40]), and the final result for the second-order energy contribution is

$$
\mathcal{E}_{2} \cong \frac{1}{2 \pi J} \iint_{S} d^{2} \mathbf{r} d^{2} \mathbf{r}^{\prime} G\left(\mathbf{r}, \mathbf{r}^{\prime}\right) E_{S}(\mathbf{m}, \mathbf{n}) E_{S}\left(\mathbf{m}, \mathbf{n}^{\prime}\right)
$$

Taking into account the cubic symmetry and computing numerically a double surface integral one can write the result of Eq. (40) as

$$
\mathcal{E}_{2} \cong \kappa \frac{L^{2} \mathcal{N}}{J_{0}}\left(m_{x}^{4}+m_{y}^{4}+m_{z}^{4}\right), \quad \kappa=0.53465
$$

where $J_{0}=z J=6 J$. This defines the large- $N$ asymptotes in Fig. 12 (right) shown by the horizontal lines.

The analytical results above is valid for particle sizes $N$ in the range

$$
1 \ll N \ll J / L
$$

The lower boundary is the applicability condition of the continuous approximation. Since the surface of a nanoparticle is made of atomic terraces separated by atomic steps, each terrace and each step with its own form of NSA [see Eq. (32)], the variation of the local NSA along the surface is very strong. Approximating this variation by a continuous function according to Eq. (35) requires pretty large particle sizes $N$. This is manifested by a slow convergence to the large- $N$ results in Fig. 12 (right). The upper boundary in Eq. (42) is the applicability condition of the linear approximation in $\psi$. For $N \gtrsim J / L$ deviations from the collinear state are strong, and the effective anisotropy of a magnetic nanoparticle cannot be introduced.

As we have seen in Eq. (41), the contribution of the surface anisotropy to the overall anisotropy of a magnetic particle scales with its volume $V \propto N^{3} \sim \mathcal{N}$. This surprising result is due to the penetration of perturbations from the surface deeply into the bulk. If a uniaxial bulk anisotropy $K_{c}$ was present in the system, perturbations from the surface would be screened at the bulk correlation length (or the domain-wall width) $\delta \sim \sqrt{J / K_{c}}$. Then for $N \gtrsim \delta$ the contribution of the surface anisotropy to the overall anisotropy would scale as the surface: $\mathcal{E}_{2} \sim\left(L^{2} / J\right) N^{2} \delta$. For not too large particles, $N \lesssim \delta$, contributions of both anisotropies to the anisotropic energy are additive and scale as the volume. If the bulk anisotropy is cubic, both contributions have the same cubic symmetry [c.f. Eqs. (4) and (41)], and the experiment should provide a 
value of the effective cubic anisotropy different form the bulk value [42]. For the uniaxial bulk anisotropy, the two contributions have different functional forms. Even if the bulk anisotropy is dominant so that the energy minima are realized for $\mathbf{m} \| \mathbf{e}_{z}$, the surface anisotropy makes the energy dependent on the azimuthal angle $\varphi$. This modifies particle's energy barrier by creating saddle points and strongly influences the process of thermal activation [44].

For small deviations from the cubic or spherical shape, i.e., for weakly elliptic or weakly rectangular particles, there should emerge a corresponding weak first-order contribution $\mathcal{E}_{1}$ that would add up with our second-order contribution. For an ellipsoid with axes $a$ and $b=a(1+\epsilon), \epsilon \ll 1$, the anisotropy energy scales with the surface, $\mathcal{E}_{1} \sim L \mathcal{N}^{2 / 3} \epsilon m_{z}^{2}[\mathrm{cf}$. Eq. (41)], so that

$$
\frac{\mathcal{E}_{2}}{\mathcal{E}_{1}} \sim \frac{L}{J} \frac{N}{\epsilon}
$$

can be large even for $L / J \ll 1$.

The Néel constant $L$ is in most cases poorly known. However, for metallic Co [45] quotes the value of surface anisotropy $-1.5 \times 10^{8} \mathrm{erg} / \mathrm{cm}^{3}$, i.e., $L \sim-10$ $\mathrm{K}$. This is much smaller than $J \sim 10^{3} \mathrm{~K}$, which makes our theory valid for particle sizes up to $N \sim J / L \sim 100$, according to Eq. (42). For this limiting size one has $\mathcal{E}_{2} / \mathcal{E}_{1} \sim 1 / \epsilon$ that is large for nearly spherical particles, $\epsilon \ll 1$.

\subsection{Surface effects on the magnetization of a nanoparticle at $T>0: M C$}

In this section, we consider more realistic model of round-shaped (spherical or ellipsoidal) nanoparticle of simple cubic or spinel crystalline structure, uniaxial or cubic anisotropy in the core, and transverse or Néel anisotropy on the surface, described by the Hamiltonian defined by Eqs. (1), (2), (4), and (3). Using various techniques explained above, we compute the magnetization, induced and intrinsic, as a function of temperature and applied magnetic field, for different values of the surface anisotropy constant and exchange coupling [4][46]. We shall mainly focus on novel features stemming from the combination of anisotropy, field and temperature effects on the magnetization.

\subsubsection{Ferromagnetic particles with Néel's surface anisotropy}

In our simulations we consider the core ferromagnetic coupling $J_{c}$ and uniaxial anisotropy $K_{c}=0.01 J_{c}$. On the surface we adopt $J_{s}=J_{c}$, while the anisotropy is given by the Néel expression (3) with constant $K_{s}=0.1 J_{c}$. We ignore the DDI for simplicity. In particular, we are interested in how anisotropy affects the superparamagnetic relation (8) that has been shown to hold at 

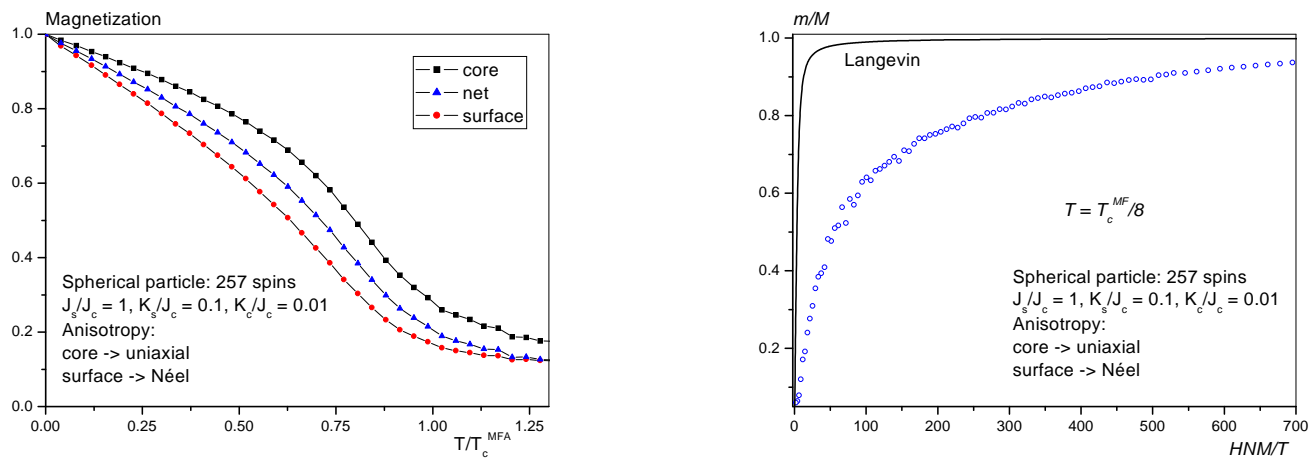

Fig. 13. Left: Core, surface and net magnetizations as functions of temperature. Right: Circles: Scaled graph for the induced magnetization $m$ as a function of $H N M / T$, where $M$ is the intrinsic magnetization. Full line: Langevin function $L(x)=\operatorname{coth} x-1 / x$.

all temperatures below $T_{c}$ for isotropic systems. As no analytical calculations are possible here, we resort to the Monte Carlo technique with global spin rotations. In Fig. 13 (left) we plot the core, surface and net magnetizations of a nanoparticle of 257 spins, as functions of temperature in zero magnetic field. These results do confirm what was obtained from the spherical model [see Fig. 1 (left)] for isotropic box-shaped systems, namely that boundary effects suppress the magnetization, and here we see that this effect is enhanced by the SA.A drastic effect of the SA is clearly seen in the field dependence of the scaled induced magnetization $m$ as shown in Fig. 13 (right) at $T=T_{c}^{M F A} / 8$. In the presence of a strong $\mathrm{SA}$ the superparamagnetic relation (8) is no longer valid, and $m / M$ strongly deviates from the Langevin function.
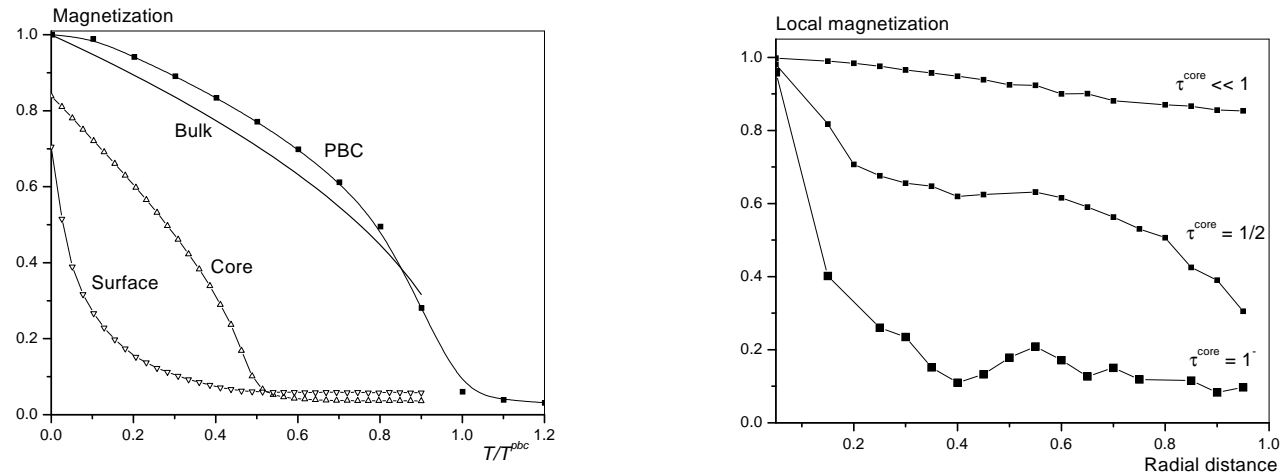

Fig. 14. Left: Temperature dependence of the surface and core magnetizations for $\mathcal{N}=3766$, magnetization of the bulk system, and that of the cube with the spinel structure and periodic boundary conditions (PBC) with $\mathcal{N}=40^{3}$. Right: Spatial variation of the net magnetization of a spherical nanoparticle of 3140 spins, as a function of the normalized particle radius, for $\tau^{\text {core }} \equiv T / T_{c}^{\text {core }} \ll 1$, and $\tau^{\text {core }}=0.5$, $\tau^{\text {core }} \simeq 1^{-}$. 


\subsubsection{Maghemite $\left(\gamma-\mathrm{Fe}_{2} \mathrm{O}_{3}\right)$ nanoparticles}

In this section we deal with the ferrimagnetic maghemite nanoparticles $(\gamma$ $\mathrm{Fe}_{2} \mathrm{O}_{3}$ ) having spinel crystalline structure, summarizing the results of [4], [46]. This time we include the DDI in the Hamiltonian of Eq. (1). The bulk anisotropy in such materials is cubic, still we are using a uniaxial anisotropy to simplify the study of effects that are of more interest to us here. We consider maghemite particles of various sizes $\left(\mathcal{N} \simeq 10^{3}-10^{5}\right.$ that correspond to a radius of 2-3.5 nm) and with the physical properties in the core (spinel crystal structure with lacuna, exchange and dipolar interactions, anisotropy constant, etc.) as those of the bulk, except that the anisotropy is taken as uniaxial. We use the TSA model with $K_{s}=0.06 \mathrm{erg} / \mathrm{cm}^{2}$. All spins in the core and on the surface are identical but interact via different couplings depending on their locus in the lattice. We assume that the exchange interactions between the core and surface spins are the same as those inside the core. Although we treat only the crystallographically "ideal" surface, we do allow for a scatter in the exchange constants on the surface. In contrast, in Refs. [12] it was assumed that all exchange interactions are the same but there was postulated the existence of a fraction of missing bonds on the surface.

In Fig. 14 (left), we see that the surface magnetization decreases more rapidly than the core magnetization as the temperature increases. Moreover, it is seen that even the (normalized) core magnetization per site does not reach its saturation value of 1 at very low temperatures, which shows that the magnetic order in the core is disturbed by the surface. This may also be due to lacuna in the spinel structure. In Fig. 14 (right) we plot the spatial evolution of the local magnetization from the center to the border of the particle, at different temperatures. At all temperatures it decreases with distance from the center. At high temperatures, the local magnetization exhibits a temperature-dependent jump, and then continues to decrease. This indicates that there is a radius within which the magnetization assumes relatively large values. This result agrees with that of [3] where this radius was called the magnetic radius.

\section{Conclusion}

We have demonstrated by different analytical and numerical methods the importance of accounting for the magnetization inhomogeneities in magnetic nanoparticles, especially in the presence of SA. The latter makes the magnetization inhomogeneous even at $T=0$ and in general modifies the relation between the intrinsic and induced magnetizations. It also changes the magnetization switching mechanism, since for large SA the particle's spins switch cluster-wise. For small SA we were able to calculate the spin canting in the particle analytically and to obtain a novel second order contribution to the 
particle's overall anisotropy. It remains to generalize this result for nonzero bulk anisotropy. Another important task is to study dynamical implications of the many-body effects in magnetic nanoparticles.

\section{References}

[1] L. Néel, C. R. Acad. Sci. Paris 228 (1949) 664; Ann. Geophys. 5 (1949) 99.

[2] W. Wernsdorfer, Adv. Chem. Phys. 118 (2001) 99.

[3] V. Wildpaner, Z. Phys. B 270 (1974) 215.

[4] H. Kachkachi et al., Eur. Phys. J. B 14 (2000) 681.

[5] E. M. Chudnovsky and J. Tejada, Macroscopic quantum tunneling of the magnetic moment, Cambridge University Press, 1998.

[6] H. W. Schumacher et al., Phys. Rev. Lett. 90 (2003) 17201; 17204.

[7] E. C. Stoner and E. P. Wohlfarth, Philos. Trans. R. Soc. London, Ser. A 240 (1948) 599; IEEE Trans. Magn. MAG-27 (1991) 3475.

[8] W. F. Brown, Phys. Rev. 130 (1963) 1677; IEEEM 15 (1979) 1196.

[9] W. T. Coffey et al., Adv. Chem. Phys. 117 (2001) 483.

[10] H. Kachkachi and D. A. Garanin, Physica A 300 (2001) 487.

[11] H. Kachkachi and M. Dimian, Phys. Rev. B 66 (2002) 174419; M. Dimian and H. Kachkachi, J. Appl. Phys. 91 (2002) 7625.

[12] R. H. Kodama et al., Phys. Rev. Lett. 77 (1996) 394; R.H. Kodama and A.E. Berkovitz, Phys. Rev. B 59 (1999) 6321.

[13] J. T. Richardson et al., J. Appl. Phys. 70 (1991) 6977.

[14] J. P. Chen et al., Phys. Rev. B 51 (1995) 11527.

[15] A. Ezzir, Propriétés Magnétiques d'une assemblée de nanoparticules: modélisation de l'aimantation, Université Paris-Sud, Orsay 1998.

[16] M. Respaud et al., Phys. Rev. B 57 (1998) 2925.

[17] E. Tronc et al., J. Mag. Mag. Mat. 221 (2000) 110.

[18] W. T. Coffey et al., Adv. Chem. Phys. 117 (2001) 483.

[19] K. Haneda, Can. J. Phys. 65 (1987) 1233.

[20] P. Prené et al., Hyperfine Int. 93 (1994) 1049.

[21] S. Mörup, J. Mag. Mag. Mat. 266 (2003) 110. 
[22] A. Aharoni, Introduction to the theory of ferromagnetism, Oxford Science Pubs. 1996.

[23] W. F. Brown, Jr., Micromagnetics, Interscience New York, 1963.

[24] V. Shilov, Effects of surface anisotropies of the ferromagnetic resonance in ferrite nanoparticles, Ph.D. thesis of University Paris VII, 1999.

[25] L. Néel, J. Phys. Radium 15 (1954) 225.

[26] H. E. Stanley, Phys. Rev. Lett. 20 (1968) 589; Phys. Rev. 176 (1968) 718.

[27] T. N. Berlin and M. Kac, Phys. Rev. 86 (1952) 821.

[28] D. A. Garanin, Z. Phys. B 102 (1997) 283; J. Phys. A 29 (1996) L257; 29 (1996) 2349; 32 (1999) 4323; Phys. Rev. E 58 (1998) 254.

[29] D. A. Garanin and V. S. Lutovinov, Solid State Commun. 50 (1984) 219.

[30] D. A. Garanin, J. Stat. Phys. 74 (1994) 275; Phys. Rev. B 53 (1996) 11593.

[31] M. E. Fisher and V. Privman, Phys. Rev. B 32 (1985) 447; Commun. Math. Phys. 103 (1986) 1986.

[32] K. Binder, in Phase Transitions and Critical Phenomena, Eds. C. Domb and J. L. Lebowitz, Academic Press, New York 1983, vol.8, pp.75-267; H. W. Diehl, ibid 1986, vol. 10, pp. 75-267.

[33] A. J. Bray and M. A. Moore, Phys. Rev. Lett. 38 (1977) 735.

[34] J. L. Cardy, J. Phys. A 16 (1983) 3617.

[35] K. Binder, in Computational methods in field theory, Eds. H. Gausterer and C. B. Lang, Springer, Berlin 1992.

[36] , D. A. Garanin, Phys. Rev. E 58 (1998) 254.

[37] M. Pleimling and W. Selke, Eur. Phys. J. B 5 (1998) 805.

[38] H. Kachkachi and D. A. Garanin, Eur. Phys. J. B 22 (2001) 291.

[39] D. A. Dimitrov and Wysin, Phys. Rev. B 50 (1994) 3077.

[40] D. A. Garanin and H. Kachkachi, Phys. Rev. Lett. 90 (2003) 65504.

[41] C. Chen, O. Kitakami, S. Okamoto, and Y. Shimada, J. Appl. Phys. 86 (1999) 2161.

[42] M. Jamet et al., Phys. Rev. Lett. 86 (2001) 4676.

[43] Y. Labaye et al., J. Appl. Phys. 91 (2002) 8715.

[44] D. A. Garanin et al, Phys. Rev. E 60 (1999) 6499.

[45] D. S. Chuang et al., Phys. Rev. B 49 (1994) 15084.

[46] H. Kachkachi et al., J. Mag. Mag. Mat. 221 (2000) 158. 